Review Article

\title{
Bacterial Biodiversity, Cold Adaptation and Biotechnological Importance of Bacteria Occurring in Antarctica
}

\author{
S SHIVAJI ${ }^{1,2, *}$, G S N REDDY ${ }^{1}$ and M K CHATTOPADHYAY ${ }^{1}$ \\ ${ }^{1}$ CSIR-Centre for Cellular and Molecular Biology, Uppal Road, Hyderabad 500 007, India \\ ${ }^{2}$ Prof. Brien Holden Eye Research Centre, L V Prasad Eye Institute. Banjara Hills, Hyderabad 500034, \\ India
}

(Received on 20 May 2016; Accepted on 24 November 2016)

\begin{abstract}
Antarctica is the coldest, iciest, windiest and driest continent and defines the limits of temperature at which life forms can survive and divide. These cold loving microorganisms are known as psychrophiles and are present in all the unique habitats of Antarctica including permafrost and ice. Their distribution and abundance varies from habitat to habitat and several new genera and species have been discovered in the icy continent. They have several strategies by which they survive and divide at low temperature such as the ability to catalyze reactions and continue metabolism with cold-tolerant enzymes; ability to maintain optimum membrane fluidity at low temperature; occurrence of specific genes required for survival at low temperatures; presence of antifreeze-activity etc.. Enzymes from psychrophilic bacteria have been found to be useful for several purposes ranging from recombinant DNA technology to food processing. The ability of cold-tolerant organisms to degrade petroleum products and other environmental pollutants highlight them as potential candidates for bioremediation in extreme cold environments. The recently reported genome sequences of a number of novel cold-tolerant isolates are likely to provide some more insights into the mechanism of bacterial cryotolerance.
\end{abstract}

Keywords: Non-Cultivable Diversity; Cultivable Diversity; Novel Species; Cold Adaptation; Antarctica

\section{Introduction}

About $85 \%$ of the Earth's biosphere is permanently exposed to temperatures below $5^{\circ} \mathrm{C}$ and these cold habitats span from the Arctic to the Antarctic, from high-mountains such as Himalayas to the deep ocean and also includes frozen soils (permafrost), glaciers and ice sheets, polar sea ice and snow (Deming and Eicken, 2007). A major fraction of this low temperature environment is represented by the deep sea which constitutes $71 \%$ of the Earth's area. Other cold environments include cold water lakes, cold soils (especially subsoils), cold deserts, and caves. All these permanently cold environments have been successfully colonized by a class of microorganisms known as psychrophiles (grow from subzero to $30^{\circ} \mathrm{C}$ ) (Morita, 1975; Helmke and Weyland, 2004; Laucks et al., 2005). Among all the cold habitats, Antarctica is unique in that it is the coldest, driest, windiest and iciest (with ice covered at an average thickness of $1.9 \mathrm{Km}$ ) of all known habitats covering approximately 5.4 million square miles in area and is thus considered as one of the most extreme habitats of the world (Vincent, 1988; Claridge and Campbell, 1977; Campbell and Claridge, 2000; Smith et al., 1992). Despite the harsh climatic conditions, along with other life forms (such as mites, ticks, seals, penguins, mosses, lichens, bacteria, yeasts, algae etc) (http:// www.globalclassroom.org/antarct6.html), psychrophilic bacteria dominate the continent and aid in nutrient recycling (Stokes and Redmond, 1966; Herbert and Bell, 1977; Tanner and Herbert, 1981; Delille and Legarde, 1974; Tanner, 1985; Voytek and Ward, 1995; Chessa et al., 2000; Kelly et al., 1978; Cavanagh et al., 1996; Denner et al., 2001; Cavicchioli and Thomas, 2000). Ekelof (1908 a and b) initiated studies on microbiology of the soil and air in Antarctica which was confirmed by Pirie (1904),

*Author for Correspondence: E-mail: shivas@ccmb.res.in; shivas@lvpei.org 
Gazert (1912), Tsiklinsky (1908) and McLean (1918 a and b). Later the emphasis was to enumerate and identify unique microorganisms from the continent of Antarctica (Boyd, 1962; Boyd and Boyd, 1962; Friedmann, 1980; Margni and Castrelos, 1963, 1965; Marshall and Ohye, 1966; Meyer et al., 1962; Pfiser and Burkholder, 1965; Tsyganov, 1970). However with the advent of culture independent identification of microorganisms based on 16S rRNA gene metagenomics a greater diversity of microbes from various habitats of Antarctica was discovered. Browsing the NCBI (http://ncbi.nlm.nih.gov) database for $16 \mathrm{~S}$ rRNA gene sequences indicated that about $600016 \mathrm{~S}$ rRNA gene sequences of bacteria from Antarctica have been deposited in the database. Of these close to 210 sequences corresponded to the novel species so far described from Antarctica (Shivaji and Reddy, 2009; Chattopadhyay et al., 2014; Reddy et al., 2016).

\section{Diversity of Antarctica as Studied by Non- cultivable Methods}

Antarctic bacterial diversity has been studied by both cultivable and non-cultivable methods (using 16S rRNA gene sequence and certain other functional genes) from various habitats including soil, cyanobacterial mats, water, sediments, sub-glacial out flow, crypto-endolithic sand stones, anoxic meromictic sediments, sediment core, ephemeral streams, ice core, geothermal vents, ornithogenic soil, penguins, sponges and intestinal microbiota of Antarctic fish (for reviews see Reddy et al., 2016; Shivaji and Reddy, 2009; Chattopadhyay et al., 2014; Bottos et al., 2014). The bacterial abundance from the above habitats of Antarctica ranged from $0.2 \times 10^{2}$ to $0.6 \times 10^{12}$ cells/ gram (Carpenter et al., 2000; Priscu et al., 1999; Karl et al., 1999; Delille and Gleizon, 2003), $0.2 \times 10^{2}$ to $10^{7}$ cells/ml (Takii et al., 1986; Franzmann et al., 1990; Lo Giudice et al., 2012) and $8 \times 10^{6}$ to $2.4 \times 10^{7}$ cells/ gram (Lanoil et al., 2009; Stibal et al., 2012) and $10^{5}$ to $10^{10}$ cells/gm (Ramsay and Stannard, 1986; Aislabie et al., 2009) for ice, water, sediment and soil, respectively. Antarctic soils are highly heterogeneous and the diversity varied with respect to soil type. For instance, Antarctic Peninsula soils were dominated by Proteobacteria (47.0\%), where as in West Antarctic soils and Transantarctic mountain soils Actinobacteria and Bacteriodetes $(42.0 \%$ and $31 \%$ repectively) (Bottos et al., 2014) were dominant and in the Victoria Land soils Actinobacteria was dominant. Interestingly members of DeinococcusThermus are the third most dominating community (18.0\%) in Transantarctic Mountain soils indicating that the bacterial communities across Antarctic soils vary significantly with geography, climate, soil physicochemical parameters and local biological influences. Further, unaffiliated clones associated with rare and unique taxa such as Verrucomicrobia and Gemmatimonadetes were also identified from Antarctic soils from Schirmacher Oasis (Shivaji et al., 2004). In addition to above phylotypes Antarctic peninsula soils, west Antarctic soils and east Antarctic soils contain several unkown phylotypes (Shivaji et al., 2004; Aislabie et al., 2006; Yergeau et al., 2007 a, b; Niederberger et al., 2008; Aislabie et al., 2009; Lee et al., 2012; Tiao et al., 2012; Bajerski and Wagner, 2013; Aislabie et al., 2013).

Limited diversity studies on Antarctic sediments from Bratina island (Sjoling and Cowan, 2003), Ardley island (Li et al., 2006), meromictic marine basin, Vestfold hills (Bowman et al., 2000), Wright Glacier (Stibal et al., 2012) revealed the dominance of phylotypes belonging to Proteobacteria, CytophagaFlavobacterium-Bacteroides, Spirochaetaceae, Actinobacteria, Acidobacteria, Gemmatimonadetes, Firmicutes, Prochlorococcus, Cyanobacteria, Deltaproteobacteria (specially the unique groups of Desulfosarcina, Syntrophus and Geobacter/Pelobacter/Desulphuromonas group), order Chlamydiales (Parachlamydiaceae), Planctomycetes and members of Spirochaetales. Carr et al. (2013) identified a total of fifty bacterial phyla from marine sediments beneath the Ross Ice Shelf and the predominant taxa reported were Actinobacteria (6.0\%), Bacteroidetes (46.0), Chloroflexi (25.0\%), Firmicutes (14.0\%), and the subphyla Beta- (20.0\%), Delta- (25.0\%) and GammaProteobacteria $(8.0 \%)$. The Betaproteobacteria were represented by only two genera Thiobacillus and Teptidiphilum and there was unusually high proportion of Chloroflexi group. Bowman et al. (2000) demonstrated that anoxic marine sediments contained more than 200 distinct phylotypes with close to $31.0 \%$ belonging to the low G+C Gram-positives. Interestingly while studying the vertical distribution of bacterial phyla in fresh water sediment in which Proteobacteria, Bacteroidetes, Actinobacteria and Firmicutes were dominant. Shivaji et al. (2011) 
identified a few clones belonging to Caldiserica group, a thermophilic candidate phylum from Antarctica.

Aquatic microbial diversity of Antarctica is limited as only $0.4 \%$ of the total ice area of Antarctica (12.3 $9106 \mathrm{~km} 2$ ) is seasonally ice free (Wilkins et al., 2013). Within this ice-free 50,850 Sq. Km area of Antarctica, the majority of bacteria belong to the phyla Proteobacteria representing 47.6\% (Alphaproteobacteria [71.5\%], Betaproteobacteria [18.8\%], Deltaproteobacteria [2.0\%] and Gammaproteobacteria [7.7\%]) with dominating genera being Rhodobacter and Sphingomonas (Huang et al., (2013). Besides, Bacteroidetes (15.1\%), Actinobacteria (14.8\%), Chloroflexi (10.2\%), Acidobacteria (6.7\%), Firmicutes (3.6\%), Gemmatimonadetes (1.3\%) and Verrucomicrobia $(0.2 \%)$ are minor components, while DeinococcusThermus, Nitrospira, Planctomycetes, and Fusobacteria were also present. The most common genera are Sphingomonas, Caulobacter, Brevundimonas, Janthinobacterium, Duganella, Polaromonas, Variovorax, Rhodoferax, Flavobacterium, Pedobacter, Prevotella, Hymenobacter and Arcicella (Wilkins et al., 2013). In general, shift in diversity was observed with depth of sediment wherein the predominance of Bacteroidetes, Betaproteobacteria and Actinobacteria in the surface are replaced by Gammaproteobacteria with depth (Archer et al., 2014). This is attributed to the tolerance to oxygen requirement of various members of the genera belonging to Gammaproteobacteria. The above observation was further supported by Kim et al., (2014) wherein deep waters contained a high proportion of unclassified Bacteria (10-20\%) along with Proteobacteria. Interestingly, the surface water contained two major clades representing Polaribacter (20 to 64\%) and uncultivated Oceanospirillaceae (7 to $34 \%$ ) while Pelagibacter increased in abundance with depth (7 to 42\%) (Kim et al., 2014). Response of bacterial community to hydrocarbon contamination also revealed a change in phylotypes profile. Alphaproteobacteria, Gammaproteobacteria, the Cytophaga-FlavobacteriumBacteroidetes were major communities but genera Psychrobacter, Arcobacter, Formosa algae, Polaribacter, Ulvibacter and Tenacibaculum were detected only in hydrocarbon contaminated water and the abundance of Sulfitobacter group was high in sea water and decreased drastically by almost 9 folds in contaminated seawater (Prabagaran et al., 2007). The variation in marine and fresh water aquatic systems was evident in Atarctica. The fresh water ecosystems are dominated by groups such as Flavobacterium, Pseudomonas and Polaromonas (Michaud et al., 2012) while Sulfitobacter, Thalassospira, members of Roseobacter, Gelidibacter, Polaribacter, Psychroflexus, Psychromonas and Pseudoalteromonas were restricted to marine waters (Prabagaran et al., 2007; Guibert et al., 2012; Lo Giudice et al., 2012).

Sea ice, in general, is a dynamic, porous matrix that harbors a network of brine pores and channels that harbor active (Junge et al., 2004; Søgaard, 2010) and diverse (Brown and Bowman, 2001; Brinkmeyer et al., 2003; Maas et al., 2012) bacterial communities. The ice-associated community contains photosynthetic, chemoautotrophic and heterotrophic bacteria beside Archaea and several other eukaryotes. Approximately $36 \%, 25 \%$ and $25 \%$ were contributed by Gammaproteobacteria, Alphaproteobacteria and Cytophaga-Flavobacterium group while members of Actinobacteria were very rarely present. Among the Gammaproteobacteria species of the genera Colwellia and Glaciecola were the most abundant and Marinobacter spp. were rare. The Alphaproteobacteria were dominated by members of the Roseobacter lineage while CF group was centered on Polaribacter group (Bowman et al., 1997; Gosink et al., 1998; Brown and Bowman, 2001; Junge et al., 2002; Brinkmeyer et al., 2003; Kuhn et al., 2014; Lanoil et al., 2009). Besides, the occurrence of communities such as Shewanella, Marinobacter, Planococcus, Alteromonas, Pseudoalteromonas, Psychrobacter, Halomonas, Pseudomonas, Hyphomonas, Sphingomonas, Arthrobacter, Planococcus, and Halobacillus were also reported (Bowman et al., 1997). The temporal community analyses indicated a shift in community wherein Paenisporosarcina was reduced by 5 folds while Bacillus increased by 4 folds. In addition, Acenetobacter and Cohnella replaced Paenibacillus and Jeotgalibacillus in a span of two years (Doyle et al., 2013). 


\section{Diversity of Antarctica as Studied by Using Functional Genes}

Besides the routine 16S rRNA based diversity, functional gene based diversity was also explored from Antarctic habitats and the genes used were basically targeted to look for the sulfate-reducing bacteria (SRB), photosynthetic bacteria, hydrocarbon degrading bacteria, chitinase and ketosynthase producing communities. Targeting the gene $d s r A$ (dissimilatory sulfite reductase), diverse group of sulfate-reducing bacteria was detected in Antarctic habitats (Karr et al., 2005). Recently, Watanabe et al. (2013) detected phylotypes, based on aprA (adenosine-5'-phosphosulfate reductase alpha subunit) belonging to the genera Thiocapsa, Sulfuricella, Desulfobacterium, Desulfofaba and Desulfotomaculum from Antarctic fresh water lakes. While more than $70 \%$ of sulfur transformation in subglacial lake sediments was contributed by two genera Sideroxydans and Thiobacillus (Purcell et al., 2014). Further, the involvement of Marinobacter, Roseovarius and Psychroflexus in marine-derived hypersaline lake in the Vestfold Hills, Antarctica, that has the highest concentration of dimethylsulfide (DMS) in a natural body of water was reported (Yau et al., 2013). The above studies indicated the dominance and involvement of Alphaproteobacteria and Betaproteobacteria in sulfate reduction and also aerobic anoxygenic photosynthesis in Antarctic habitats. In addition, based on Polycyclic aromatic hydrocarbon ring-hydroxylating dioxygenase (PAHRHD) gene, diverse groups belonging to Proteobacteria, Actinobacteria, Verrucomicrobia, Bacteroidetes, Firmicutes, Chloroflexi, Gemmatimonadetes, Cyanobacteria, Chlorobium, and Acidobacteria were reported from Antarctic soils and sediments. Among these the genera Terrabacter, Mycobacterium, Diaphorobacter belonging to Gram-positives and Sphingomonas and Burkholderia of Gram-negatives were predominant (Muangchinda et al., 2014). While 14 distinct phylotypes representing the genera Rhodococcus, Mycobacterium, Nocardioides, Terrabacter and Bacillus were identified from Antarctic sediments based on PAH-dioxygenases (Marcos et al., 2009). Diversity studies based on alkane monooxygenases (Alk) genes from a sediment sample indicated the predominance of unique genes that are differentially distributed between the two sites, the Admiralty Bay and King George Island of Peninsula Antarctica. The gene sequences identified the genera Silicibacter, Gordonia, Prauserella, Nocardioides, Rhodococcus, Nocardia farcinica, Pseudomonas, Acidisphaer and Alcanivorax (Kuhn et al., 2009). Besides, differential response of bacterial communities to hydrocarbon contamination was studied by Prabagaran et al. (2007). Studies based on chitinase genes (chi67, chi69,chiA, chiB, chiF) revealed the occurrence of Janthinobacterium, Stenotrophomonas, Cytophaga, Streptomyces and Norcardiopsis as the abundant genera from a 1600 year old sediment obtained from Ardley Island, Antarctica (Xiao et al., 2005). The phylogenetic analysis based on ketosynthase (KS) identified Proteobacteria, Firmicutes, Planctomycetes, Cyanobacteria, Actinobacteria, some uncultured symbiotic bacteria and five independent clades. Most of the identified KS showed below $80 \%$ identities at the AA level to their closest match revealing the great diversity and novelty of ketosynthase genes in Antarctic sediments (Zhao et al., 2008). Based on gene pufM (a gene coding for photosynthetic pigment-binding protein) 33 unique phylotypes related to Rubrivivax, Acidiphilum, Rhodoferax and Roseateles were detected from Antarctic sediments (Karr et al., 2003; Stibal et al., 2012). While studying the diversity of $p s b A$ gene from Lake Bonney, unique phylotypes of genera Nannochloropsis, Ochromonas and Isochrysis were identified (Kong et al., 2014). The amoA based diversity studies indicated the dominance of only two genera, the Nitrosospira and Nitrosomonas, as the ammonium oxidizing community in Antarctic habitats (Magalhães et al., 2014).

\section{Diversity of Antarctica as Studied by Cultivable Methods}

The cultivable bacterial diversity was explored extensively from various habitats such as soil (Miwa, 1975; Yi and Chun, 2006; Ruckert, 1985; Shivaji et al., 1988; 1989a; 1989b; 1991; Wery et al., 2003; Bozal et al., 2007), water (Lo Giudice et al., 2012; Michaud et al., 2012; Soller et al., 2000; Labrenz et al., 2000; Cristóbal et al., 2011), algal mats (Reddy et al., 2000; 2002; 2003a; 2003b; 2004; Van Trappen et al., 2002; Spring et al., 2003; Peeters et al., 2011), ice (Shivaji et al., 2004; Antibus et al., 2012; Bowmen et al., 1998) and sediments (Shivaji et al., 2011; Yu et al., 2011) and reviewed by Shivaji and Reddy (2009) and 
Chattopadyay et al. (2014) from Antarctica. Among the habitats, water, soil and mats are more diverse compared to ice and sediments as evident from the absence and abundance of various communities. In all the habitats the most dominating communities are Proteobacteria, Cytophaga-FlavobacteriumBacteriodetes, Actinobacteria and Firmicutes and communities belonging to Deinococcus-thermus and Spirochaetes are restricted, interestingly, only to soil (Hirsch et al., 2004; Antibus et al., 2012; Franzmann \& Dobson, 1992; Chattopadyay et al., 2014). Among the Proteobacteria, Gammaproteobacteria leads in abundance in soil (3\%) and water (0.6\%) while members of Epsilonproteobacteria were absent (Reddy et al., 2016).

Relative percentage occurrence of cultivable bacteria from Antarctica indicated that the soils are dominated by Firmicutes (35\%), Actinobacteria (25\%) and Proteobacteria (18\%), water is inhabited by Proteobacteria (42\%), CytophagaFlavobacterium-Bacteriodetes (31\%) and Actinobacteria (23\%), algal mats are rich in Proteobacteria (40\%), Firmicutes (28\%) and Cytophaga-Flavobacterium-Bacteriodetes (24\%), ice is abundant in Proteobacteria (48\%), CytophagaFlavobacterium-Bacteriodetes (21\%) and Firmicutes (19\%) and finally the sediments communities are composed of Proteobacteria (50\%) and Actinobacteria (29\%) (Reddy et al., 2016). The above statistics clearly indicate that Proteobacteria is ubiquitously present in all the Antarctic habitats. Close to 500 cultivable bacteria isolated from Antarctica represent the above major groups of Proteobacteria, Cytophaga-FlavobacteriumBacteriodetes, Actinobacteria and Firmicutes represented by 172 genera (38 novel genera) and 209 novel species.

\section{Novel Species From Antarctica}

Till date close to 209 bacterial species have been described based on polyphasic taxonomy (except, Desulfovibrio of Deltaproteobacteria, Hymenobacter species of CFB group and a few species of Carnobacterium and Clostridium of Firmicutes), from various habitats of Antarctica and these novel species belonged to the phyla Alphaproteobacteria, Betaproteobacteria, Gammaproteobacteria, Deltaproteobacteria,
Epsilonproteobacteria, CFB, Firmicutes, Actinobacter and Deinococcus-Thermus. The relative frequency of the above isolates at the phyla level is shown in Fig. 1 and Table 1 (Bottos et al., 2014; Chattopadhyay et al., 2014; Reddy et al., 2016). The most common feature of the novel species of Antarctica is their psychrophilic growth wherein they grow from zero or subzero temperatures to $30^{\circ} \mathrm{C}$ but not beyond. However, a few species like Alicyclobacillus pohliae $\left(42-60^{\circ} \mathrm{C}\right)$ (Imperio et al., 2008), Aneurinibacillus terranovensis $\left(20-55^{\circ} \mathrm{C}\right)$, (Allan et al., 2005), Anoxybacillus amylolyticus (45$\left.65^{\circ} \mathrm{C}\right)$ (Poli et al., 2006), Bacillus fumrioli $\left(25-55^{\circ} \mathrm{C}\right)$ (Logan et al., 2000), Brevibacillus levickii $\left(15-55^{\circ} \mathrm{C}\right)$ (Allan et al., 2005), Paenibacillus cineris $\left(8-50^{\circ} \mathrm{C}\right)$ and Paenibacillus cookie $\left(15-50^{\circ} \mathrm{C}\right)($ Logan et al., 2004) are moderately thermophilic and interestingly all these species were isolated from soil. The physiological characteristics with respect to the extracellular enzymes indicate approximately $79 \%$ of novel species secrete the enzyme phosphatase followed by protease (48.7\%), lipase (48.5\%), amylase (42\%), $\beta$-glactosidase (32\%) and urease (20\%) (Fig. 2). Further, $68 \%$ of novel species described so far

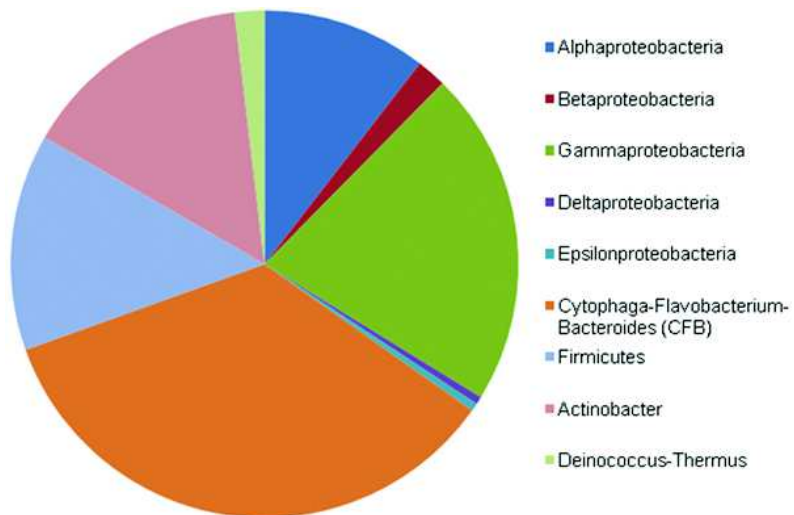

Fig. 1: Relative percentage of novel species described from various habitats of Antarctica

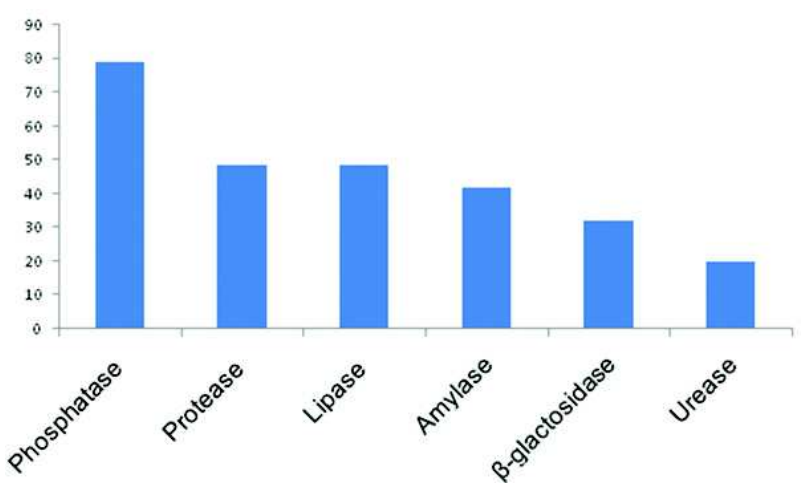

Fig. 2: Production of extracelluar enzymes by novel species from Antarctica 


\begin{tabular}{|c|c|c|c|c|c|c|c|c|c|c|c|c|c|c|c|c|c|c|c|}
\hline \multicolumn{2}{|c|}{ S.No. } & \multirow{2}{*}{$\begin{array}{l}\text { Name of the species } \\
\text { Alphaproteobacteria }\end{array}$} & \multirow[t]{2}{*}{ A } & \multirow[t]{2}{*}{$\mathrm{C}$} & \multirow[t]{2}{*}{$\mathrm{L}$} & \multirow[t]{2}{*}{$\mathrm{P}$} & \multirow[t]{2}{*}{$\mathrm{U}$} & \multirow[t]{2}{*}{ BG } & \multirow[t]{2}{*}{$\mathrm{Ph}$} & \multirow[t]{2}{*}{$\mathrm{Pi}$} & \multirow[t]{2}{*}{ FA } & \multirow[t]{2}{*}{ MK } & \multirow[t]{2}{*}{$\mathrm{DA}$} & \multirow[t]{2}{*}{$\mathrm{G}+\mathrm{C}$} & \multirow[t]{2}{*}{$\mathrm{Ca}$} & \multirow[t]{2}{*}{$\mathrm{O}$} & \multirow[t]{2}{*}{$\mathrm{H}$} & \multirow[t]{2}{*}{ Lipids } & \multirow[t]{2}{*}{$\begin{array}{l}\text { 16S rRNA gene } \\
\text { Acc. No }\end{array}$} \\
\hline & & & & & & & & & & & & & & & & & & & \\
\hline 1 & 1 & $\begin{array}{l}\text { Antarctobacter } \\
\text { heliothermus* }\end{array}$ & - & - & - & + & ND & ND & ND & $\mathrm{BY}$ & $18: 1$ & Q10 & ND & 62.3 & + & + & water & PG, PC & Y11552 \\
\hline 2 & 2 & $\begin{array}{l}\text { Constrictibacter } \\
\text { antarcticus* }^{*}\end{array}$ & ND & ND & + & ND & ND & ND & + & - & $18: 1$ & Q10 & ND & 69.8 & + & - & Rock & ND & AB510913 \\
\hline 3 & 3 & Loktanella fryxellensis* & - & ND & - & - & - & + & + & PG & $18: 1 \omega 7 \mathrm{c}$ & ND & ND & 66 & + & + & Mats & ND & AJ582225 \\
\hline 4 & 4 & Loktanella salsilacus & - & ND & - & - & - & + & + & Biege & $18: 1 \omega 7 \mathrm{c}$ & ND & ND & 60 & + & + & Mats & ND & AJ440997 \\
\hline 5 & 5 & Loktanella vestfoldensis & - & ND & + & - & + & + & + & Pink & $18: 1 \omega 7 \mathrm{c}$ & ND & ND & 63 & + & + & Mats & ND & AJ582226 \\
\hline 6 & 6 & $\begin{array}{l}\text { Polymorphobacter } \\
\text { multimanifer* }\end{array}$ & ND & ND & - & - & - & - & + & $\mathrm{Br}$ & 17:1ù6c & Q10 & ND & 68 & + & + & Rock & PG, PE, SPL & AB649056 \\
\hline 7 & 7 & $\begin{array}{l}\text { Pseudorhodobacter } \\
\text { antarcticus }\end{array}$ & - & ND & + & - & - & - & + & Pink & $18: 1 \omega 7 \mathrm{c}$ & Q10 & ND & 57.1 & + & + & Sediment & PG, PC & FJ196030 \\
\hline 8 & 8 & $\begin{array}{l}\text { Pseudorhodobacter } \\
\text { collinsensis }\end{array}$ & - & + & - & + & + & + & $+C$ & Cream & $18: 1 \omega 7 \mathrm{c}$ & Q10 & ND & 61 & + & + & Ice cap & PC, PE, PG & KM978076 \\
\hline 9 & 9 & $\begin{array}{l}\text { Pseudorhodobacter } \\
\text { psychrotolerans }\end{array}$ & - & ND & - & + & - & + & $+C$ & Cream & $18: 1 \omega 7 \mathrm{c}$ & Q10 & ND & 60.1 & + & + & Soil & PC, PG, UL & KT163920 \\
\hline 10 & 10 & $\begin{array}{l}\text { Puniceibacterium } \\
\text { antarcticum* }\end{array}$ & - & ND & - & - & - & - & + & PR & $18: 1 \omega 7 \mathrm{c}$ & ND & ND & 60.7 & + & + & Water & PC, PE, PG & KP136797 \\
\hline 11 & 11 & $\begin{array}{l}\text { Rhodoligotrophos } \\
\text { appendicifer* }\end{array}$ & ND & ND & - & - & - & - & - & Red & $16: 0$ & Q9 & ND & 61.1 & + & + & Water & PE, PG & AB617575 \\
\hline 12 & 12 & $\begin{array}{l}\text { Robiginitomaculum } \\
\text { antarcticum* }\end{array}$ & ND & ND & + & - & - & + & +1 & Rusty & $18: 1 \omega 7 \mathrm{c}$ & Q10 & ND & 60.3 & + & - & Water & ND & EF495229 \\
\hline 13 & 13 & Roseibaca ekhonensis* & - & ND & - & - & ND & ND & ND & Red & $18: 1 \omega 7 \mathrm{c}$ & Q10 & ND & 61 & + & + & Water & PC, PE, PG, DPG & AJ605746 \\
\hline 14 & 14 & $\begin{array}{l}\text { Roseicitreum } \\
\text { antarcticum* }\end{array}$ & - & ND & - & - & + & + & ND & Pink & $18: 1 \omega 7 \mathrm{c}$ & Q10 & ND & 63.3 & + & + & Sediment & PC, PE, PG & FJ196006 \\
\hline 15 & 15 & $\begin{array}{l}\text { Roseisalinus } \\
\text { antarcticus* }\end{array}$ & - & ND & - & - & ND & ND & ND & Rose & $18: 1 \omega 7 \mathrm{c}$ & Q10 & mDap & 67 & + & + & Water & ND & AJ605747 \\
\hline 16 & 16 & Roseovarius antarcticus & - & ND & - & - & - & - & + & PY & $18: 1 \omega 7 \mathrm{c}$ & Q10 & ND & 61 & + & + & Bone & PC, PE, PG, DPG & KM347966 \\
\hline 17 & 17 & Roseovarius tolerans* & - & ND & + & - & ND & ND & ND & Red & $18: 1$ & Q10 & ND & 64 & + & + & Water & PC, PE, PG, DPG & Y11551 \\
\hline 18 & 18 & Sphingomonas aerolata & - & ND & - & - & ND & + & + & $\mathrm{O}$ & $18: 1 \omega 7 \mathrm{c}$ & Q10 & ND & 65.4 & + & + & Water & PC, PE, PG, DPG, SPL & AJ429240 \\
\hline 19 & 19 & $\begin{array}{l}\text { Sphingomonas } \\
\text { aurantiaca }\end{array}$ & - & ND & - & - & ND & + & + & $\mathrm{O}$ & $18: 1 \omega 7 \mathrm{c}$ & Q10 & ND & 65 & + & + & Water & PC, PE, PG, DPG, SPL & AJ429236 \\
\hline
\end{tabular}




\begin{tabular}{|c|c|c|c|c|c|c|c|c|c|c|c|c|c|c|c|c|c|c|c|}
\hline 20 & 20 & Sphingomonas faeni & - & ND & - & - & ND & + & + & $\mathrm{O}$ & $18: 1 u ̀ 7 c$ & Q10 & ND & 63 & + & + & Water & PC, PE, PG, DPG, SPL & AJ429239 \\
\hline 21 & 21 & Staleya guttiformis* & - & ND & ND & - & ND & ND & ND & - & $18: 1 u ̀ 7 c$ & Q10 & ND & 56 & - & + & Water & PC, PE, PG & Y16427 \\
\hline 22 & 22 & Sulfitobacter brevis & - & ND & ND & - & ND & ND & ND & - & $18: 1 u ̀ 7 c$ & Q10 & ND & 58 & - & + & Water & PC, PE,D PG & Y16425 \\
\hline \multirow[t]{2}{*}{23} & 23 & Thalassospira lohafexi & - & ND & - & + & - & - & + & - & $18: 1 u ̀ 7 c$ & Q10 & ND & 53 & + & + & Water & PE, PG & GU584152 \\
\hline & & Betaproteobacteria & & & & & & & & & & & & & & & & & \\
\hline 24 & 1 & $\begin{array}{l}\text { Actimicrobium } \\
\text { antarcticum* } 2 / 4\end{array}$ & ND & ND & + & - & - & - & + & - & 16:1ù7c & Q8 & ND & 65.9 & + & + & Water & PE, PG, UL & HQ699437 \\
\hline 25 & 2 & $\begin{array}{l}\text { Herbaspirillum } \\
\text { psychrotolerans }\end{array}$ & + & ND & ND & + & + & ND & ND & - & 16:1ù7c & Q8 & ND & 62.5 & + & - & Soil & PE, PG, DPG & JN390675 \\
\hline 26 & 3 & Polaromonas vacuolata* & - & ND & + & - & + & ND & ND & - & $16: 1 u ̀ 7 c$ & ND & ND & 52 & + & + & Water & ND & U14585 \\
\hline \multirow[t]{2}{*}{27} & 4 & Rhodoferax antarcticus & - & ND & ND & ND & ND & ND & NDF & Peach & ND & ND & ND & 61.5 & & & Mat & ND & AF084947 \\
\hline & & Gammaproteobacteria & & & & & & & & & & & & & & & & & \\
\hline 28 & 1 & Alteromonas stellipolaris & + & ND & + & + & - & + & ++ & $\mathrm{Br}$ & $16: 0$ & ND & ND & 45 & + & + & Water & ND & AJ295715 \\
\hline 29 & 2 & Glaciecola Polaris & + & ND & + & - & - & + & + & - & $16: 0$ & ND & ND & 44 & + & + & Water & ND & AJ293820 \\
\hline 30 & 3 & $\begin{array}{l}\text { Granulosicoccus } \\
\text { antarcticus } * *\end{array}$ & - & ND & + & + & - & + & - & - & 16:1ù7c & Q8 & ND & 58 & + & + & Water & ND & EF495228 \\
\hline 31 & 4 & Granulosicoccus marinus & - & - & + & + & - & + & + & - & $18: 1 u ̀ 7 c$ & Q8 & ND & 61 & + & + & Water & ND & KF146345 \\
\hline 32 & 5 & Hahella Antarctica & ND & ND & - & - & - & - & + & $\mathrm{Cr}$ & $16: 1 u ̀ 7 c$ & Q9 & ND & 56.4 & + & - & Water & ND & EF495227 \\
\hline 33 & 6 & $\begin{array}{l}\text { Halomonas } \\
\text { alkaliantarctica }\end{array}$ & - & ND & ND & - & - & ND & ND & - & $18: 1$ & Q9 & ND & 55 & - & + & Water & PE, PG, DPG & AJ564880 \\
\hline 34 & 7 & Halomonas glaciei & - & ND & - & - & - & ND & - & - & $18: 1$ & Q8 & ND & - & + & + & Water & ND & AJ431369 \\
\hline 35 & 8 & Lysobacter oligotrophicus & + & - & + & + & ND & + & ND & DB & i15:0 & Q8 & ND & 66 & + & + & Water & PE, PG, DPG & AB694977 \\
\hline 36 & 9 & Marinobacter antarcticus & - & ND & + & - & - & - & + & $\mathrm{Br}$ & $16: 1 u ̀ 7 c$ & ND & ND & 55.8 & + & + & Water & PE, PG, DPG & FJ196022 \\
\hline 37 & 10 & Marinobacter guinea & - & ND & + & - & - & - & + & - & 16:1ù7c & Q9 & ND & 57.1 & + & + & Sediment & ND & AM503093 \\
\hline 38 & 11 & Marinobacter maritimus & - & ND & + & - & + & - & + & $\mathrm{Cr}$ & $18: 1 u ̀ 7 c$ & Q9 & ND & 58 & + & + & Water & ND & AJ704395 \\
\hline 39 & 12 & Marinomonas Polaris & - & ND & - & - & ND & + & + & - & $18: 1 u ̀ 7 c$ & ND & ND & 41.2 & + & - & Water & PE, PG & AJ833000 \\
\hline 40 & 13 & Marinomonas ushuaiensis & + & ND & - & - & - & ND & + & - & 18:1ù7c & ND & ND & 43.6 & + & - & Water & ND & AJ627909 \\
\hline 41 & 14 & Methylosphaera hansonii* & ND & ND & ND & ND & - & ND & - & $\mathrm{Y}$ & 16:1ù7c & ND & ND & 46 & + & + & Sediment & ND & U67929 \\
\hline 42 & 15 & Neptunomonas Antarctica & - & ND & - & - & - & - & + & - & 16:1ù7c & Q8 & ND & 46 & + & + & Sediment & ND & FJ713802 \\
\hline 43 & 16 & Oleispira lenta & - & ND & + & - & - & - & + & - & $16: 1 u ̀ 7 c$ & ND & ND & 43 & - & + & Water & ND & EU980447 \\
\hline 44 & 17 & Oleispira antarctica* & - & ND & + & - & - & ND & ND & - & 16:1ù7c & ND & ND & 42 & + & + & Water & ND & AJ426420 \\
\hline 45 & 18 & $\begin{array}{l}\text { Pseudoalteromonas } \\
\text { antarctica }\end{array}$ & - & ND & + & + & - & - & + & - & 16:1ù7c & ND & ND & 42 & + & + & Mud & ND & X98336 \\
\hline
\end{tabular}




\begin{tabular}{|c|c|c|c|c|c|c|c|c|c|c|c|c|c|c|c|c|c|c|c|}
\hline 46 & 19 & $\begin{array}{l}\text { Pseudoalteromonas } \\
\text { prydzensis }\end{array}$ & + & ND & + & + & + & - & + & - & $16: 1 \omega 7 \mathrm{c}$ & ND & ND & 39 & + & + & Ice & ND & U85855 \\
\hline 47 & 20 & Pseudomonas antarctica & - & - & + & - & + & - & + & - & $18: 1$ & ND & ND & 61 & + & + & Mat & ND & AJ537601 \\
\hline 48 & 21 & Pseudomonas deceptionensis & & ND & - & - & + & - & - & - & $16: 1$ & ND & ND & 53.8 & + & + & Sediment & PE, PG, DPG & GU936597 \\
\hline 49 & 22 & $\begin{array}{l}\text { Pseudomonas } \\
\text { extremaustralis }\end{array}$ & - & ND & ND & - & - & - & ND & - & $16: 1 \omega 7 \mathrm{c}$ & ND & ND & ND & + & + & Water & ND & AF405328 \\
\hline 50 & 23 & Pseudomonas guinea & - & ND & + & - & - & - & + & - & $16: 0$ & ND & ND & 58.5 & + & + & Soil & ND & AM491810 \\
\hline 51 & 24 & Pseudomonas meridiana & - & - & + & - & + & - & - & - & $16: 0$ & ND & ND & 63 & + & + & Mat & ND & AJ537602 \\
\hline 52 & 25 & Pseudomonas prosekii & - & - & - & + & - & ND & ND & - & ND & ND & ND & ND & + & + & Soil & ND & JN814372 \\
\hline 53 & 26 & Pseudomonas proteolytica & - & - & + & + & - & - & - & - & $18: 1$ & ND & ND & 58 & + & + & Mat & ND & AJ537603 \\
\hline 54 & 27 & Psychrobacteradeliensis & - & - & - & + & - & - & - & - & $18: 1 \omega 9 \mathrm{c}$ & ND & ND & 45 & + & + & Water & ND & AJ539105 \\
\hline 55 & 28 & Psychrobacter aquaticus & - & - & + & - & + & - & + & - & $16: 1$ & Q8 & ND & 43.6 & + & + & Mat & PE, PG, DPG & AJ584833 \\
\hline 56 & 29 & Psychrobacterfozii & - & ND & - & - & + & ND & + & - & $18: 1 \omega 9 \mathrm{c}$ & ND & ND & 44 & + & + & Sediment & ND & AJ430827 \\
\hline 57 & 30 & Psychrobacter frigidicola & - & ND & - & - & - & - & - & - & $18: 1 \omega 9 c$ & $\mathrm{ND}$ & ND & 42 & + & + & Water & ND & AJ609556 \\
\hline 58 & 31 & Psychrobacter luti & - & ND & + & - & - & ND & + & - & $18: 1 \omega 9 \mathrm{c}$ & ND & ND & 45 & + & + & Soil & ND & AJ430828 \\
\hline 59 & 32 & Psychrobacter nivimaris & - & ND & + & - & ND & ND & ND & - & $18: 1 \omega 9 \mathrm{c}$ & ND & ND & 42 & + & + & Org.Mate & ND & AJ313425 \\
\hline 60 & 33 & Psychrobacter proteolyticus & - & ND & + & + & + & - & + & - & $18: 1 \omega 9 \mathrm{c}$ & ND & ND & 43.6 & + & + & Water & ND & $\mathrm{AJ} 272303$ \\
\hline 61 & 34 & Psychrobacter salsus & - & ND & - & - & - & - & - & - & $18: 1 \omega 9 \mathrm{c}$ & ND & ND & 44 & + & + & Water & ND & AJ539104 \\
\hline 62 & 35 & Psychrobacter urativorans & - & ND & - & - & + & - & - & - & $18: 1 \omega 9 \mathrm{c}$ & ND & ND & 46 & + & + & Water & ND & AJ609555 \\
\hline 63 & 36 & Psychrobacter vallis & - & - & + & - & - & - & + & - & $18: 1 \omega 9 \mathrm{c}$ & Q8 & ND & 46 & + & + & Mat & PE, PG, DPG & AJ584832 \\
\hline 64 & 37 & Psychromonas antarcticus* & - & ND & ND & + & ND & ND & ND & - & $16: 1 \omega 7 \mathrm{c}$ & Q8 & ND & 43 & + & + & Soil & ND & Y14697 \\
\hline 65 & 38 & Psychromonas kaikoae & - & ND & ND & + & ND & ND & ND & - & $16: 1 \omega 7 \mathrm{c}$ & Q8 & ND & 44 & + & + & Sediment & PE, PG, DPG & AB052160 \\
\hline 66 & 39 & Saccharospirillum impatiens & $s^{*}+$ & ND & - & + & ND & ND & ND & - & $18: 1$ & Q8 & mDap & 55 & + & + & Water & PE, PG, DPG & AJ315983 \\
\hline 67 & 40 & Shewanella livingstonensis & - & ND & + & + & - & + & + & - & $16: 1 \omega 7 \mathrm{c}$ & MK7 & ND & 41 & + & + & Water & PE, PG, DPG & AJ300834 \\
\hline 68 & 41 & Shewanella frigidimarina & - & ND & + & + & - & - & + & - & $16: 1 \omega 7 \mathrm{c}$ & $\mathrm{ND}$ & ND & 43 & + & + & Ice & ND & U85903 \\
\hline 69 & 42 & Shewanella gelidimarina & - & ND & + & + & - & - & + & - & $16: 1 \omega 7 \mathrm{c}$ & ND & ND & 48 & + & + & Ice & ND & U85907 \\
\hline 70 & 43 & Shewanella vesiculosa & - & $\mathrm{ND}$ & + & + & - & + & + & - & $16: 1 \omega 7 \mathrm{c}$ & Q7 & ND & 42 & + & + & Sediment & ND & AM980877 \\
\hline 71 & 44 & Zhongshania antarctica* & - & ND & - & - & - & - & ND & - & $17: 1 \omega 8 \mathrm{c}$ & ND & ND & 52.5 & + & + & Ice & ND & FJ889619 \\
\hline 72 & 45 & $\begin{array}{l}\text { Zhongshania guokunii } \\
\text { Deltaproteobacteria }\end{array}$ & - & ND & - & - & - & - & ND & - & $17: 1 \omega 8 \mathrm{c}$ & ND & ND & 51.8 & + & + & Water & ND & FJ889678 \\
\hline 73 & 1 & Desulfovibrio lacusfryxellens & se & & ot wel & char & acteriz & & & & & & & & & & Water & & DQ767889 \\
\hline
\end{tabular}




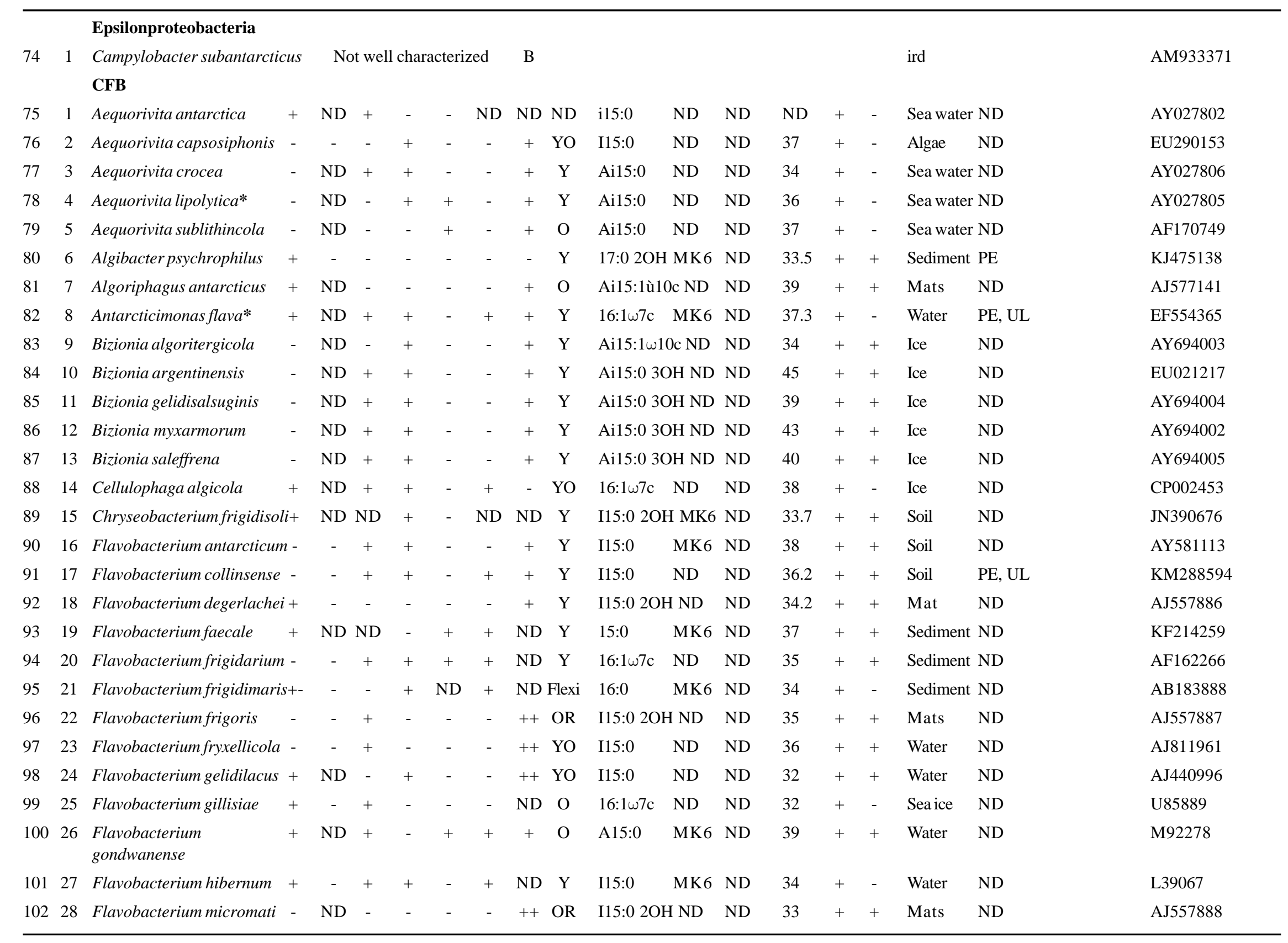




\begin{tabular}{|c|c|c|c|c|c|c|c|c|c|c|c|c|c|c|c|c|c|c|c|}
\hline $\begin{array}{l}103 \\
\text { psych }\end{array}$ & $\begin{array}{l}29 \\
\text { hroli }\end{array}$ & $\begin{array}{l}\text { Flavobacterium } \\
\text { Iimnae }\end{array}$ & + & - & - & - & - & - & ++ & $\mathrm{Y}$ & \multicolumn{2}{|c|}{$\mathrm{I} 15: 02 \mathrm{OH}$} & ND & ND & 34.5 & + & + Water & ND & AJ585428 \\
\hline 104 & 30 & Flavobacterium segetis & + & - & + & - & - & + & + & $\mathrm{O}$ & $16: 1 \omega 7 \mathrm{c}$ & MK6 & ND & 41 & + & + & Soil & ND & AY581115 \\
\hline 105 & 31 & Flavobacterium salegens & + & ND & + & + & - & + & + & $\mathrm{Y}$ & $16: 1 \omega 7 \mathrm{c}$ & MK6 & ND & 41 & + & + & Water & ND & M92279 \\
\hline 106 & 32 & Flavobacterium tegetincola & - & - & + & - & - & - & ND & $\mathrm{Y}$ & $16: 1 \omega 7 \mathrm{c}$ & ND & ND & 32 & + & - & Mud pool & IND & U85887 \\
\hline 107 & 33 & Flavobacterium weaverense & + & - & + & - & + & - & + & $\mathrm{Y}$ & $16: 1 \omega 7 \mathrm{c}$ & MK6 & ND & 37 & + & + & Soil & ND & AY581114 \\
\hline 108 & 34 & Gelidibacter algens* & + & $\mathrm{ND}$ & + & + & - & - & + & $\mathrm{Y}$ & A15:0 & ND & ND & 38 & + & - & Sea ice & ND & U62914 \\
\hline 109 & 35 & Gelidibacter gilvus & - & ND & + & - & - & + & - & $\mathrm{Y}$ & A15:0 & ND & ND & 39 & + & - & Sea ice & ND & AF001369 \\
\hline 110 & 36 & Gelidibacter salicanalis & - & ND & - & + & + & + & + & $\mathrm{Y}$ & a15:1w10 & $\mathrm{c} N \mathrm{ND}$ & ND & 42 & + & - & Sea ice & ND & AY694009 \\
\hline 111 & 37 & Gillisia hiemivivida & + & ND & + & + & + & - & + & $\mathrm{Y}$ & A15:0 & ND & ND & 34 & + & + & Ice core & ND & AY694006 \\
\hline 112 & 38 & Gillisia illustrilutea & - & ND & - & - & - & - & - & $\mathrm{Y}$ & 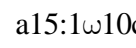 & $\mathrm{c} N \mathrm{ND}$ & ND & 32 & + & + & Ice core & ND & AY694008 \\
\hline 113 & 39 & Gillisia limnaea* & - & ND & - & + & - & - & + & $\mathrm{Y}$ & $17: 02 \mathrm{OH}$ & $\mathrm{H}$ ND & ND & 38 & + & + & Mats & ND & AJ440991 \\
\hline 114 & 40 & Gillisia sandarakina & + & ND & + & + & - & - & + & $\mathrm{O}$ & i16:0 & ND & ND & 36 & + & + & Ice core & ND & AY694007 \\
\hline 115 & 41 & Hymenobacter algoricola & & t well & 1 chara & cterize & & & & $\mathrm{RP}$ & $16: 1 \omega 7 \mathrm{c}$ & ND & ND & ND & - & + & Gl. water & ND & EU155009 \\
\hline 116 & 42 & Hymenobacter antarcticus & & & & & & & & $\mathrm{RP}$ & $16: 1 \omega 7 \mathrm{c}$ & ND & ND & ND & - & + & Gl. Water & ND & EU155012 \\
\hline 117 & 43 & Hymenobacter elongates & & & & & & & & $\mathrm{RP}$ & $\mathrm{I} 17: 1$ & ND & ND & ND & - & + & Gl. Water & ND & GQ454797 \\
\hline 118 & 44 & Hymenobacterfastidiosus & & & & & & & & $\mathrm{RP}$ & $16: 1 \omega 7 \mathrm{c}$ & ND & ND & $\mathrm{ND}$ & - & + & Gl. Water & ND & EU155015 \\
\hline 119 & 45 & Hymenobacter glaciei & & & & & & & & $\mathrm{RP}$ & $16: 1 \omega 7 \mathrm{c}$ & ND & ND & ND & - & + & Gl. water & ND & GQ454806 \\
\hline 120 & 46 & $\begin{array}{l}\text { Hymenobacter } \\
\text { roseosalivarius* }\end{array}$ & + & - & + & + & - & ND & + & $\mathrm{RP}$ & $16: 1 \omega 7 \mathrm{c}$ & MK7 & ND & 56 & + & + & Soil & PE & Y18833 \\
\hline 121 & 47 & Kordia Antarctica & + & - & - & - & - & - & + & $\mathrm{Y}$ & $16: 1 \omega 9 \mathrm{c}$ & MK6 & ND & 35 & - & - & Sea water & PE, UL & JX456458 \\
\hline 122 & 48 & Lacinutrix copepodicola* & - & ND & + & + & - & - & + & Y & I15:0 & MK6 & ND & 32 & + & + & Sea ice & $\mathrm{PE}$ & AY694001 \\
\hline 123 & 49 & Lacinutrix jangbogonensis & - & ND & + & + & - & - & + & $\mathrm{Y}$ & I15:0 & MK6 & ND & 37 & + & + & Sea ice & PE & KF977035 \\
\hline 124 & 50 & Leeuwenhoekiella aequorea & + & - & + & + & - & + & + & Y & $17: 1 \omega 9 \mathrm{c}$ & MK6 & ND & 36 & + & + & Sea ice & ND & AJ278780 \\
\hline 125 & 51 & Leeuwenhoekiella polynyae+ & +- & - & + & - & + & + & Y I & 17:0 2 & $\mathrm{OH}$ & MK6 & ND & 39 & + & + & Water & PE, UL & KM101107 \\
\hline 126 & 52 & Lewinella Antarctica & + & - & - & + & - & + & + & $\mathrm{O}$ & $16: 1 \omega 7 \mathrm{c}$ & MK7 & ND & 50 & + & - & Water & ND & EF554367 \\
\hline 127 & 53 & Maribacter antarcticus & + & ND & + & - & - & - & + & $\mathrm{O}$ & I15:0 & MK6 & ND & 37 & + & + & Algae & $\mathrm{PE}$ & EU512921 \\
\hline 128 & 54 & Muricauda Antarctica & - & $\mathrm{ND}$ & - & + & - & + & + & $\mathrm{Br}$ & $\mathrm{I} 17: 03 \mathrm{O}$ & HMK6 & ND & 45 & + & + & Sea water & $\mathrm{PE}$ & JN166984 \\
\hline 129 & 55 & Nonlabens antarcticus & - & - & - & + & - & - & + & $\mathrm{O}$ & Ai15:0 & MK6 & ND & 38 & + & + & Gl . core & PE & DQ660393 \\
\hline 130 & 56 & Pedobacter ardleyensis & + & ND & - & - & - & + & + & Red & I15:0 & MK7 & ND & 40 & + & + & Soil & PE, Sphingo lipid & KJ631640. \\
\hline 131 & 57 & Polaribacter filamentus & + & - & ND & + & - & - & ND & Soh & $\mathrm{I} 15: 02 \mathrm{OH}$ & & ND & ND & 32 & + & -Sea wate & er ND & U73726 \\
\hline 132 & 58 & Polaribacter franzmannii & + & - & ND & + & - & + & ND & $\mathrm{O}$ & $\mathrm{I} 15: 03 \mathrm{OH}$ & & ND & ND & 32 & + & + Sea ice & ND & U14586 \\
\hline
\end{tabular}




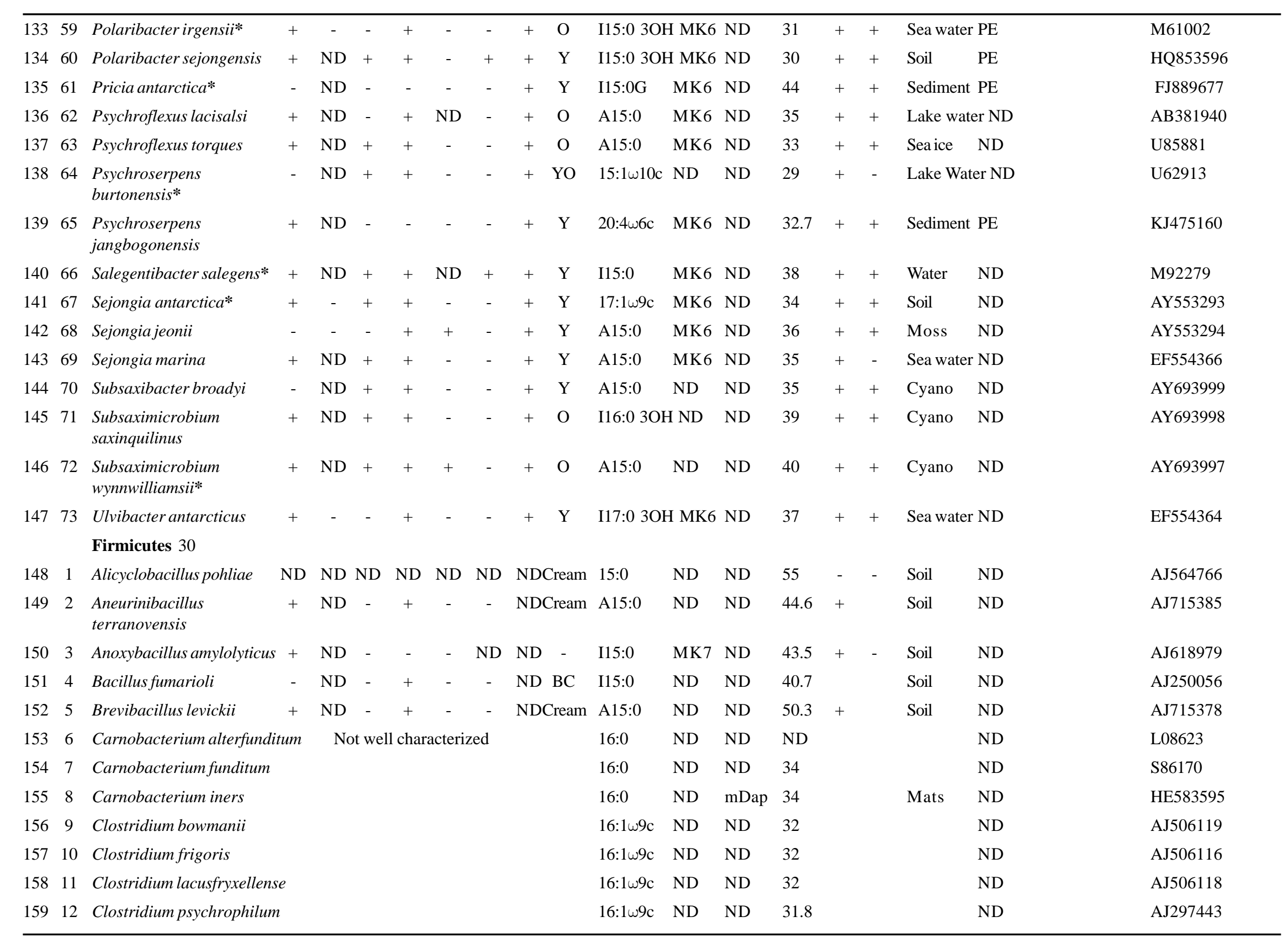




\begin{tabular}{|c|c|c|c|c|c|c|c|c|c|c|c|c|c|c|c|c|c|c|c|}
\hline 160 & 13 & Clostridium schirmacherens & & & & & & & & & $15: 0$ & ND & ND & 24 & & & & ND & AM114453 \\
\hline 161 & 14 & Clostridium vincentii & & & & & & & & & $16: 0$ & ND & ND & 33 & & & & ND & X97432 \\
\hline 162 & 15 & $\begin{array}{l}\text { Exiguobacterium } \\
\text { antarcticum }\end{array}$ & + & & - & + & - & + & + & $\mathrm{O}$ & $16: 0$ & MK7 & ND & 48.5 & + & + & Soil & $\begin{array}{l}\text { PG, DPG, PS, PI, } \\
\text { PE, UL }\end{array}$ & DQ019164 \\
\hline 163 & 16 & Exiguobacterium soli & + & & - & + & - & + & + & $\mathrm{Y}$ & $15: 0$ & MK7 & ND & 47 & + & & Soil & PG, DPG, PS, PI & AY864633 \\
\hline 164 & 17 & Exiguobacterium undae & + & & - & + & - & + & + & $\mathrm{O}$ & $16: 0$ & MK7 & ND & 48 & + & + & Soil & $\begin{array}{l}\text { PG, DPG, PS, PI, } \\
\text { PE, UL }\end{array}$ & DQ019165 \\
\hline 165 & 18 & Jeotgalicoccus pinnipedialis & $s \mathrm{ND}$ & ND & ND & + & ND & - & - & - & $15: 0$ & MK7 & ND & 38.6 & + & + & & PG, DPG, PI & AJ 251530 \\
\hline 166 & 19 & Paenibacillus antarcticus & + & ND & + & - & + & - & ND & - & a15:0 & ND & ND & 40.7 & + & + & Sediment & ND & AJ605292 \\
\hline 167 & 20 & Paenibacillus. cineris & ND & ND & ND & - & - & + & ND & - & a15:0 & ND & ND & 51.5 & + & + & Soil & ND & AJ575658 \\
\hline 168 & 21 & Paenibacillus cookie & ND & ND & ND & - & - & + & ND & $\mathrm{Y}$ & a15:0 & ND & ND & 51.6 & + & + & Soil & ND & $\mathrm{AJ} 250317$ \\
\hline 169 & 22 & Paenibacillus darwinianus & + & ND & - & - & ND & ND & ND & - & a15:0 & MK7 & ND & 55.6 & + & - & Soil & PG, DPG, PE & KF264455 \\
\hline 170 & 23 & Paenibacillus wynni & + & ND & ND & - & - & + & ND & - & a15:0 & ND & ND & 44.6 & & & Soil & & AJ633647 \\
\hline 171 & 24 & Planococcus antarcticus & - & ND & + & + & - & + & - & $\mathrm{O}$ & a15:0 & MK7,8 & ND & 41.5 & + & - & Mat & PG, DPG, PE & AJ314745 \\
\hline 172 & 25 & Planococcus maitriensis & - & ND & - & + & - & + & - & $\mathrm{O}$ & a15:0 & MK7,8 & ND & 39 & + & + & Mat & ND & AF500007 \\
\hline 173 & 26 & Planococcus mcmeekinii & - & ND & - & + & ND & ND & ND & $\mathrm{O}$ & a15:0 & MK7,8 & ND & 35 & + & - & Brine & ND & AF041791 \\
\hline 174 & 27 & Planococcus psychrophilus & - & ND & + & + & - & + & - & $\mathrm{O}$ & a15:0 & MK7,8 & ND & 44.5 & + & & Mat & PG, DPG, PE & AJ314746 \\
\hline 175 & 28 & Psychrosinus fermentans & & t well & chara & cteri & & + & + & W & & & & & & & ater & ND & DQ767881 \\
\hline 176 & 29 & Sporosarcina Antarctica & - & ND & & - & - & - & + & LY & a15:0 & MK7 & ND & 39.2 & + & + & Soil & ND & EF154512 \\
\hline 177 & 30 & $\begin{array}{l}\text { Sporosarcina } \\
\text { macmurdoensis }\end{array}$ & + & ND & - & + & - & - & + & - & a15:0 & MK7 & ND & 44 & + & - & Mat & ND & AJ514408 \\
\hline 178 & & Actinobacteria 6/31 & & & & & & & & & & & & & & & & & \\
\hline 179 & 1 & Arthrobacter antarcticus & + & $\mathrm{ND}$ & - & - & + & - & + & $\mathrm{Y}$ & a15:0 & MK8 & \multicolumn{2}{|c|}{ Lys-Glu 68} & + & + & Sediment & DPG, PE & AM931709 \\
\hline 180 & 2 & Arthrobacter ardleyensis & - & - & + & + & ND & ND & ND & $\mathrm{Y}$ & a15:0 & MK8 & \multicolumn{2}{|c|}{ Lys-Ala-Glu } & 59.5 & + & - Sediment & ND & AJ551163 \\
\hline 181 & 3 & Arthrobacter cryotolerans & - & ND & ND & + & - & - & + & - & a15:0 & $\mathrm{MK} \mathrm{H}_{2}$ & \multicolumn{2}{|c|}{ Lys-Glu } & 64.5 & + & - Soil & PG & GQ406812 \\
\hline 182 & 4 & Arthrobacter flavus & + & ND & - & - & - & ND & ND & $\mathrm{Y}$ & a15:0 & $\mathrm{MK} 9 \mathrm{H}_{2}$ & \multicolumn{2}{|c|}{ Lys-Thr-Ala 3} & 65 & + & - Mat & PE, PG, DPG & AB537168 \\
\hline 183 & 5 & Arthrobacter gangotriensis & - & ND & - & + & + & - & + & $\mathrm{Y}$ & a15:0 & MK8 & \multicolumn{2}{|c|}{ Lys-Glu } & 66 & + & + Soil & ND & AJ606061 \\
\hline 184 & 6 & Arthrobacter kerguelensis & - & ND & - & $?$ & + & - & + & Y & a15:0 & MK8 & \multicolumn{2}{|c|}{ Lys-Glu } & 58 & + & + Sea water & ND & AJ606062 \\
\hline 185 & 7 & Arthrobacter livingstonensis & $i s-$ & ND & ND & - & + & ND & ND & $\mathrm{W}$ & a15:0 & $\mathrm{MK} 9 \mathrm{H}_{2}$ & \multicolumn{2}{|c|}{ Lys-Thr-Ala } & 64.7 & + & - Soil & PG & GQ406811 \\
\hline 186 & $\begin{array}{c}8 \\
\text { hilus }\end{array}$ & Arthrobacter psychrochitini- & & + & + & - & - & ND & ND & $\mathrm{Y}$ & a15:0 & $\mathrm{MK} \mathrm{H}_{2}$ & \multicolumn{2}{|c|}{ Lys-Glu } & 58.5 & + & - Penguin & ND & AB588633 \\
\hline 187 & 9 & Arthrobacter roseus & - & ND & - & + & - & - & + & $\mathrm{R}$ & a15:0 & $\mathrm{MK} 9 \mathrm{H}_{2}$ & \multicolumn{2}{|c|}{ Lys-Thr-Ala 3} & 69 & + & -Mat & PE, PG, DPG & $\mathrm{AJ} 278870$ \\
\hline 188 & 10 & Barrientosiimonas humi* & - & - & - & + & - & ND & ND & PY & a17:0 & ${\mathrm{MK} 8 \mathrm{H}_{4}}$ & \multicolumn{2}{|c|}{ Lys-Ser-Asp } & 68.4 & + & -Soil & PE, PG, DPG, UL & JF346171 \\
\hline
\end{tabular}




\begin{tabular}{|c|c|c|c|c|c|c|c|c|c|c|c|c|c|c|c|c|c|c|c|}
\hline 189 & 11 & $\begin{array}{l}\text { Curtobacterium } \\
\text { psychrophilum* }\end{array}$ & + & ND & ND & - & ND & ND & ND & - & a15:0 & MK10 & \multicolumn{2}{|l|}{$\mathrm{Dab}$} & 65 & + & -Soil & PG, DPG, UL & D45058 \\
\hline 190 & 12 & Friedmanniella antarctica* & + & ND & + & - & + & ND & ND & $\mathrm{O}$ & a15:0 & $9 \mathrm{H} 4$ & \multicolumn{2}{|c|}{ Lys-Gly } & 73 & + & -Sand stone & PG, DPG, PI, UL & Z78206 \\
\hline 191 & 13 & Friedmanniella lacustris & - & ND & ND & + & - & ND & ND & $\mathrm{O}$ & a15:0 & $9 \mathrm{H} 4$ & mDap & 73 & + & + & Water & ND & AJ132943 \\
\hline 192 & 14 & Kocuria Polaris & + & ND & + & - & - & - & - & $\mathrm{O}$ & a15:0 & $7 \mathrm{H} 2$ & \multicolumn{2}{|c|}{ Lys-Ala 3} & 72.5 & + & + Mat & PG, DPG, PI & AJ 278868 \\
\hline 193 & 15 & Leifsonia antarctica & - & ND & - & + & - & + & + & $\mathrm{Y}$ & a15:0 & MK10 & Dab & - & + & + & Sediment & PG, DPG, PE & AM931710 \\
\hline 194 & 16 & Leifsonia aurea & - & ND & - & - & - & + & - & $\mathrm{Y}$ & a15:0 & MK11 & $\mathrm{Dab}$ & 64 & + & - & Mat & ND & AJ438586 \\
\hline 195 & 17 & Leifsonia psychrotolerans & + & ND & - & - & - & ND & ND & $\mathrm{Y}$ & a15:0 & MK10 & $\mathrm{Dab}$ & 64.5 & + & - & Soil & PG, DPG & GQ406810 \\
\hline 196 & 18 & Leifsonia rubra & - & ND & - & - & - & + & - & $\mathrm{R}$ & a15:0 & MK11 & Dab & 66 & + & - & Mat & ND & AJ438585 \\
\hline 197 & 19 & $\begin{array}{l}\text { Marisediminicola } \\
\text { antarctica* }\end{array}$ & - & ND & - & - & - & + & - & $\mathrm{Y}$ & a15:0 & MK10 & Orn & 67 & + & - & Sediment & PG, DPG & GQ496083 \\
\hline 198 & 20 & Micrococcus antarcticus & + & ND & - & - & - & ND & ND & $\mathrm{Y}$ & a15:0 & MK 8 & Lys & 64.4 & + & - & Soil & ND & EF154512 \\
\hline 199 & 21 & $\begin{array}{l}\text { Micromonospora } \\
\text { endolithica }\end{array}$ & + & - & + & + & ND & ND & \multicolumn{3}{|c|}{ NDOrangeI16:0 } & MK10 & mDap & 70 & + & - & \multicolumn{2}{|c|}{ Sand stone PE, PI, DPG } & AJ560635 \\
\hline 200 & 22 & $\begin{array}{l}\text { Modestobacter } \\
\text { multiseptatus* }\end{array}$ & + & ND & - & - & - & ND & + & Pink & $18: 0$ & MK9H2 & mDap & 69.9 & + & - & Soil & PE, PG, DPG, PI & Y18646 \\
\hline 201 & 23 & $\begin{array}{l}\text { Nesterenkonia } \\
\text { lacusekhoensis }\end{array}$ & - & ND & - & - & ND & ND & ND & $\mathrm{Y}$ & a15:0 & MK7 & Lys & 66.1 & + & - & Water & PC, PG, DPG & AJ290397 \\
\hline 202 & 24 & Nocardioides antarcticus & - & ND & + & + & - & - & $+\mathrm{C}$ & Cream & i16:0 & MK8H4 & mDap & 66.7 & + & - & Sediment & PG, DPG, UL3 & KM347967 \\
\hline 203 & 25 & Nocardioides aquaticus & + & ND & ND & + & ND & ND & ND & - & I16:1 & MK8H4 & mDap & 69 & + & - & Water & ND & X94145 \\
\hline 204 & 26 & Nocardiopsis fildesensis & - & ND & - & - & - & ND & ND & - & i16:0 & MK9H4 & mDap & 76.8 & + & & Soil & PC, PG, PME & FJ853144 \\
\hline 205 & 27 & Pseudonocardia antarctica & + & ND & - & + & + & + & $+\mathrm{B}$ & Brown & I16:0 & MK8H4 & mDap & 71 & + & + & Moraine & PC, PE, PI, PG, DPG & AJ576010 \\
\hline 206 & 28 & Rhodoglobus vestalii* & ND & ND & ND & - & - & + & + & Red & a15:0 & MK12 & Orn & 62 & + & - & Water & ND & AJ459101 \\
\hline 207 & 29 & Sanguibacter antarcticus & - & ND & - & - & - & - & - & $\mathrm{Y}$ & a15:0 & MK9H4 & ND & 69.5 & + & - & Sea Sand & ND & EF211071 \\
\hline 208 & 30 & Streptomyces fildesensis & - & ND & + & ND & - & ND & - & YG & I16:0 & MK9H6 & mDap & 70 & + & - & Soil & PE, PI, DPG, PME & DQ408297 \\
\hline \multirow[t]{2}{*}{209} & 31 & Streptomyces hypolithicus & - & - & ND & + & - & ND & ND & - & ND & ND & $\mathrm{mDap}$ & ND & ND & ND & Quartz & ND & EU196762 \\
\hline & & Deinococcus Thermus & & & & & & & & & & & & & & & & & \\
\hline 210 & 1 & Deinococcus antarcticus & + & ND & + & + & ND & + & + & Pink & \multicolumn{2}{|c|}{ 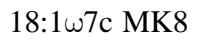 } & L-Orn & 63.1 & + & - & Soil & ND & KC494323 \\
\hline 211 & 2 & Deinococcus frigens & + & ND & ND & + & - & + & ND & $\mathrm{P}-\mathrm{O}$ & \multicolumn{2}{|c|}{$16: 1 \omega 9 \mathrm{c}$ MK8 } & L-Orn & 65.5 & + & + & Soil & ND & AJ585981 \\
\hline 212 & 3 & Deinococcus saxicola & + & ND & ND & + & - & - & ND & P-R & \multicolumn{2}{|c|}{ 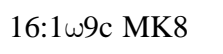 } & L-Orn & 54.4 & + & + & Soil & ND & AJ585984 \\
\hline 213 & 4 & Deinococcus marmoris & - & - & ND & + & - & + & ND & P-R & \multicolumn{2}{|c|}{$16: 1 \omega 9 \mathrm{c}$ MK8 } & L-Orn & 62.8 & + & + & Soil & ND & AJ585986 \\
\hline
\end{tabular}

*, Genus; **, Family; A, amylase; C, cellulase; L, lipase; P, protease; U, urease; BG, Beta-galactosidase; Ph, phosphatase; Ca, catalse; O, oxidase; Pig, pigment (o, orange; y, yellow; r, red; cr, cream; w, white); MK, quinine type; FA; Fatty acid methyl ester major; DA, diamino acid in peptidoglycan; GC, G+C content of the DNA; H, habitat (S, soil; Sd, sediment; Wf, fresh water; Ws, sea water; I, ice). ND = not determined; '+' = Present, '-' = Absent 
are pigmented, indicating that pigmentation is a common phenomenon in Antarctic bacteria. Pigment is known to be involved in stress adaptation to low temperatures (Jaganadham et al., 1996; Jaganadham et al., 2000; Chattopadyay et al., 1997).

Alphaproteobacteria contributed $10.5 \%$ of total novel species isolated so far and the 23 novel species belonged to 16 genera of which 12 were novel genera described from Antarctic habitats (Table 1). Six of the novel species contained Bacterial chlorophyll (Liu et al., 2014; Labrenz et al., 1999; Labrenz et al., 2000; Labrenz et al., 2005; Labrenz et al., 2009; Yu et al., 2011) and could exhibit photoautotrophic type of growth. Majority of the species produced Alkaline phosphatase and â-galactosidase but none of them secreted amylase while only 3 and 5 species exhibited the activity for protease and lipase, indicating that this group is responsible for the availability of phosphate in Antarctic habitats. The presence of pigment is a common phenomenon in all except in case of Constrictibacter antarcticus (Yamada et al., 2011). All the species contained their respective generic characteristics and the major fatty acid was $C_{18: 1} \omega 7 \mathrm{c}$ and the respiratory quinine was Q10.

Unlike Alphaproteobacteria, only very few species of Betaproteobacteria were isolated and the reason could be the inadequate information on conducive media conditions. So far four novel species belonging to 4 different genera were reported out of which 2 were novel genera. The genus Polaromonas vacuolata (Irgens et al., 1996) possessed intracellular gas vesicles. The genus Rhodoferax antarcticus contained bacteriochlorophyll (Madigan et al., 2000). All the species possessed their respective generic characteristics and the major fatty acid and respiratory quinone was $\mathrm{C}_{16: 1} \omega 7 \mathrm{c}$ and $\mathrm{Q} 8$ respectively.

Species belonging to Betaproteobacteria were the second largest group explored from Antarctic habitats. So far close to 44 novel species belonging to 18 genera were described of which 6 were novel genera. The genera Oleispira (Yakimov et al., 2003) and Methylosphaera (Bowman et al., 1997) were endemic to Antarctica. Further, novel family, Granulosicoccaceae, was reported from Antarctic water (Lee et al., 2007). Majority of species produce phosphatase (70\%) and lipase (64\%) while enzymes protease $(37.5 \%)$, urease $(23.7 \%)$, $\beta$-galactosidase
(22\%) and amylase $(16.7 \%)$ are produced by less than $50 \%$ of the novel species (Table 1). Some of the novel species were interesting wherein Pseudomonas prydzensis produces chitinase (Bowman et al., 1998), Oleispira antarctica can degrade hydrocarbons (Yakimov et al., 2003), cells of species Saccharospirillum are microaerophilic (Labrenz et al., 2003), Methylosphaera hansonii is a methanotroph (Bowman et al., 1997) and Alteromonas stellipolaris produces buds (Van Trappen et al., 2004).

CFB group of bacteria dominated various habitats of Antarctica (Bottos et al., 2014) and constitute $35 \%$ of novel species from Antarctica. Interestingly all the novel species isolated were pigmented and yellow pigmented bacteria were the most common. So far 73 novel species belonging to CFB group were described and they belonged to 27 genera out of which 12 were novel genera. In addition, species belonging to the genera Antarcticimonas, Pricia, Sejongia and Subsaximicrobium were endemic to Antarctica. Among all the bacterial phyla from Antarctica, CFB group plays an important role in nutrient recycling as they produce phosphatase $(91.5 \%)$, protease $(64.7 \%$, lipase $(57.6 \%)$, amylase $(58.8 \%), \beta$-galactosidase $(30.3 \%)$ and urease $(15.4 \%)$. Species of the genus Polaribacter exhibited filamentous morphology while members of Gelidibacter, Psychroflexus and Subsaximicrobium exhibited gliding motility compared to other members of the phylum. Further, Flavobacterium frigidimaris, $F$. gillisiae and $F$. gondwanense were found to be rich in chitinase production. The yellow pigment found in majority of the species of CFB group is a carotenoid pigment and pigment involvement in low temperature adaptation was well established (Jaganadham et al., 2000). The occurrence of pigment could be an important characteristic for the dominance of CFB members in Antarctic habitats compared to other group.

Novel species belonging to Firmicutes or Low $\mathrm{G}+\mathrm{C}$ Gram-positive group contributed 13.8\% (29 species) to the total unique species from Antarctica and 12 genera were the most common. The most common habit was soil but cyanobacterial mats also contained members of this group. Though most of the species were psychrophilic, this group also contained few moderately thermophilic species, 
Alicyclobacillus pohliae $\left(42-60^{\circ} \mathrm{C}\right)$ (Imperio et al., 2008), Aneurinibacillus terranovensis $\left(20-55^{\circ} \mathrm{C}\right)$, (Allan et al., 2005), Anoxybacillus amylolyticus (45$65^{\circ} \mathrm{C}$ ) (Poli et al., 2006), Bacillus fumrioli $\left(25-55^{\circ} \mathrm{C}\right)$ (Logan et al., 2000), Brevibacillus levickii $\left(15-55^{\circ} \mathrm{C}\right)$ (Allan et al., 2005), Paenibacillus cineris $\left(8-50^{\circ} \mathrm{C}\right)$ and Paenibacillus cookie $\left(15-50^{\circ} \mathrm{C}\right)($ Logan et al., 2004). Some members of this group were unique wherein Anoxybacillus amylolyticus produce significant quantities of EPS and amylase (Poli et al., 2006), Carnobacterium species produces lactic acid (Franzmann et al., 1991) and Clostridium schirmacherense secretes proteases (Alam et al., 2006). Further, members of Exiguobacterium possess a variety of phospholipids in their cell wall wherein they contained PE, PG, PI, PS, DPG in addition to some unknown lipids (Frühling et al., 2002; Chaturvedi et al., 2008).

Close to 31 novel species representing 16 genera of Actinobacteria were reported from various habitats of Antarctica (Table 1). Out of the 16 genera, six novel genera were described of which three genera Barrientosiimonas (Lee et al., 2013), Marisediminicola (Li et al., 2010) and Rhodoglobus (Sheridan et al., 2003) were endemic to Antarctica. Some of the novel species are unique wherein Arthrobacter cryotolerans (Ganzert et al., 2011) produces $\mathrm{H}_{2} \mathrm{~S}$, Arthrobacter psychrochitiniphilus (Wang et al., 2009) secretes cold active extracellular chitinase and Sanguibacter antarcticus (Hong et al., 2008) makes melanin. Interestingly, extracellular restriction endonuclease activity was detected in Modestobacter multiseptatus (Mevs et al., 2000). Majority of Actinobacterial species from Antarctica were pigmented indicating the role of the pigment in their survival at low temperatures. Some of the species possess unique lipids in their cell wall wherein PME (phosphatidyl methylethanolamine) and PMI (2,6,10,15,19-pentamethylicosane) are synthesized by Nocardiopsis fildesensis (Xu et al., 2014) and Streptomyces fildesensis ( $\mathrm{Li}$ et al., 2011) respectively. Among all the species Leifsonia rubra (0 to 18) (Reddy et al., 2003b) and Rhodoglobus vestalii (-2 to 21) (Sheridan et al., 2003) are unique in having the true psychrophilic growth temperature.

The coldest and driest environmental conditions persisting in Antarctica also supported the inhabitation of desiccation and radiation resistant bacterial communities such as Deinococcus. So far, four novel species of the genus Deinococcus were explored and they are radiation resistant. All the species were psychrophilic and are capable of growing from 9 to $18^{\circ} \mathrm{C}$ while Deinococcus antarcticus is a mesophile with a growth temperature range of 20 to $40^{\circ} \mathrm{C}$. All the novel species are pink to red colored with Lornithine as the cell wall diamino acid. All the species possessed unique and unidentified lipids belonging to phosphoglycolipids, glycolipids, phospholipids and lipids. These exceptional lipids can be explored for the further industrial applications and may also have a role in desiccation and radiation tolerance.

\section{Some Unique Features of Bacteria from Extreme Environments}

A majority of bacterial isolates from Antarctica shared many common features with respect to growth wherein the maximum growth temperature was below $30^{\circ} \mathrm{C}$ with very few exceptions such as Alicyclobacillus pohliae, Aneurinibacillus terranovensis, Anoxybacillus amylolyticus, Bacillus thermantarcticus and Brevibacillus levickii (Lama et al., 1996; Allan et al., 2005; Poli et al., 2006; Imperio et al., 2008). Earlier studies also revealed that the $\mathrm{G}+\mathrm{C}$ content of $16 \mathrm{~S}$ rRNA gene correlated with the growth temperature in prokaryotes. An elongation of helix 17 was observed in five out of eight true psychrophilic species of the genus Rhodoglobus (An et al., 2010) and Leifsonia rubra (Reddy et al., 2003b) whose growth temperature was below $20^{\circ} \mathrm{C}$. Occurrence of $16 \mathrm{~S}$ rDNA operons with psychrotolerant signatures, the transitions from $\mathrm{C}$ and $\mathrm{G}$ to $\mathrm{T}$ and $\mathrm{A}$, higher uracil content and $\mathrm{G}: \mathrm{U}$ mismatches from mesophilic to psychrotolerant, was reported in the strains of Bacillus (Prüss et al., 1999; Lauro et al., 2007). Further swollen sporangia were observed in psychrophilic strains of the genus Bacillus compared to their nearest mesophilic counterparts (Reddy et al., 2008; Abd El-Rahman et al., 2002; Larkin and Stokes, 1967; Priest et al., 1988). Recently, Reddy et al., (2015) identified occurrence of an additional aromatic amino acid, proline, in the CspA protein of Kocuria polaris and assisting in adaptation to low temperature.

\section{Cold Adaptation of Antarctica Bacteria}

Various survival mechanisms at extreme low temperatures have been reported in Antarctica 
bacteria and reviewed earlier (Finegold, 1986; Ray et al., 1998; Shivaji et al., 2007; Shivaji and Prakash, 2010; Singh et al., 2014; Chattopadhyay et al., 2014). Differential phosphorylation of lipopolysaccharides (low phosphorylation at low temperature) occurs in Pseudomonas syringae, an Antarctic psychrophilic bacterium, to modulate the permeability of outer membrane (Ray et al., 1994a). In addition, a temperature dependent hosphorylation and dephosphorylation was also demonstrated in Pseudomonas syringae wherein a $65 \mathrm{kDa}$ protein was found to be phosphorylated at low temperature while the $30 \mathrm{kDa}$ protein gets phosphorylated at high temperatures (Ray et al., 1994b). Further, several cold active enzymes have been studied in Antarctic bacteria and demonstrated the higher catalytic activity at low temperatures (Ray et al., 1992). Several extracellular enzymes have been characterized from Antarctic bacteria which include a protease (Ray et al., 1992), ribonuclease (Reddy et al., 1994), alkaline phosphatase (Chattopadhyay et al., 1995; Rina et al., 2000), subtilisin (Davail et al., 1994), beta-lactamase (Feller et al., 1997), citrate synthase (Russell et al., 1998), RNA polymerase (Uma et al., 1999), DNA ligase (Georlette et al., 2000), $\beta$-galactosidase (Hoyoux et al., 2001) and lipase (de Pascale et al., 2008; Ali et al., 2013). All above enzyme were catalytically active at low temperature and were heatlabile. The flexibility in activity at low temperature for enzymes from Antarctic bacteria were due to their unique structural features wherein the enzymes exhibited fewer residues of proline or arginine, decrease in hydrophobic residues and disulfide bonds and increase in polar amino acids (Gerday et al., 2000; Metpally and Reddy 2009; D'Amico et al., 2002).

The survival of Antarctic bacteria was also due to the homeoviscous adaptation of membrane fluidity. At low temperatures the membrane fluidity was maintained by changing the fatty acid composition wherein the unsaturated fatty acids, short chained and branched fatty acids are synthesized in response to low temperature (Chintalapati et al., 2004). Further, the cold inducible desaturases that convert saturated fatty acids to unsaturated fatty acids were also responsible (Chintalapati et al., 2006; Chintalapati et $a l .$, 2007) for adaptation of Antarctic bacteria. In addition, the role of cis-trans isomerase was also implicated in maintaining the membrane fluidity in Antarctic bacteria (Kiran et al., 2005). Further, the increased syntheses of polar carotenoids were observed in maintaining the homeovisous status of the membranes (Jagannadham et al., 1991; Jagannadham et al., 1996; Chattopadhyay et al., 1997).

Besides several genes and promoters involved in cold adaptation of Antarctic bacteria were explored. An amino acyltransferase (Sundareswaran et al., 2010), trmE coding for GTPase (Singh et al., 2009), hutU operon (Janiyani and Ray, 2002), rpoS (Jovcic et al., 2008), RecBCD (Pavankumar et al., 2010) gene were known to be up regulated during the cold growth. In addition several cold inducible promoters resposnsible for regulation of genes at low temperature were identified (Duilio et al., 2004).

Our group has recently reported the genome sequence of a number of psychrophiles from different cold habitats like Antarctica (Sreenivas et al., 2014; Reddy et al., 2013; Kumar et al., 2013 a), Arctic (Shivaji et al., 2013a; 2013b; Kumar et al., 2013 b), Himalayan glaciers (Reddy et al., 2014) and Stratosphere (Shivaji et al., 2012). A comparative genome analysis of Csps in the psychrophilic Sphingobacterium antarcticum, Oceanisphaera arctica and Exiguobacterium indicum indicated that CspA was present in all of them, Csp C, D, E and G were present in at least one of the 3 isolates whereas Csp B and I were absent (unpublished). The implications of this observation with respect to cold adaptation warrant further studies. Comparative genome analysis of psychrophiles besides providing information on the role of specific genes in cold adaptation would promise insight into the adaptive response of bacteria to other stressors (viz high salt, high and low $\mathrm{pH}$ ).

\section{Biotechnological Potential of Antarctica Bacteria}

Cold-active and/or thermolabile enzymes obtained from cold-tolerant organisms are of immense biotechnological importance. A thermolabile phosphatase, from an Antarctic bacterium has been used for restriction enzyme digestion, dephosphorylation, enzyme inactivation, and ligation or end-labelling. In addition cold-active proteases are suitable for food processing and in leather industries and are also useful in laundries for removal of stains. Cold-adapted bacteria are capable of degrading hydrocarbons (Margesin and Schinner, 2001; Powell 
et al., 2006; Shukor et al., 2009; Timmis et al., 2010) but some hydrocarbons like asphalt are recalcitrant in nature and resist microbial degradation (Kimes et $a l ., 2014)$. Microorganisms having the capacity to degrade PCBs have also been isolated from both the Arctic and Antarctic regions (Master and Mohan 1998; De Dominico et al., 2004). Several strains are also known to produce lipases useful in lipase-mediated biodiesel production (Moreno and Rojo, 2014).

\section{References}

Abd El-Rahman H A, Fritze D, Spröer C and Claus D (2002) Two novel psychrotolerant species, Bacillus psychrotolerans sp. nov. and Bacillus psychrodurans sp. nov., which contain ornithine in their cell walls Int J Syst Evol Microbiol 52 2127-2133

Aislabie J M, Chhour K L, Saul D J, Miyauchi S, Ayton J, Paetzold R F and Balks M R (2006) Dominant bacteria in soils of Marble Point and Wright Valley, Victoria Land, Antarctica Soil Biol Biochem 38 3041-3056

Aislabie J, Jordan S, Ayton J, Klassen J L, Barker G M and Turner S (2009) Bacterial diversity associated with ornithogenic soil of the Ross Sea region, Antarctica Can J Microbiol 55 21-36

Aislabie J M, Lau A, Dsouza M, Shepherd C, Rhodes P and Turner S J (2013) Bacterial composition of soils of the Lake Wellman area, Darwin Mountains, Antarctica Extremophiles 17 775-786

Alam S I, Dixit A, Reddy G S, Dube S, Palit M, Shivaji S and Singh L (2006) Clostridium schirmacherense sp. nov., an obligately anaerobic, proteolytic, psychrophilic bacterium isolated from lake sediment of Schirmacher Oasis, Antarctica Int J Syst Evol Microbiol 56 715-20

Ali M S, Ganasen M, Rahman R N, Chor A L, Salleh A B and Basri M (2013) Cold-adapted RTX lipase from Antarctic Pseudomonas sp. strain AMS8: isolation, molecular modeling and heterologous expression Protein J 32 317-25

Allan R N, Lebbe L, Heyrman J, De Vos P, Buchanan C J and Logan N A (2005) Brevibacillus levickii sp. nov. and Aneurinibacillus terranovensis sp. nov., two novel thermoacidophiles isolated from geothermal soils of northern Victoria Land, Antarctica Int J Syst Evol Microbiol 55 1039-1050

\section{Conclusion}

Microorganisms are a bioresource and the workhorses of biotechnology. Further extremophiles like the ones that survive under freezing temperatures are all the more important due to their ability to carry out biological processes under extreme freezing temperatures. This review focuses on the bacterial biodiversity of different habitats of Antarctica and explores their capability to survive under freezing condition and highlights their biotechnological potential.

An S Y, Xiao T and Yokota A (2010) Reclassification of Leifsonia aurea to the genus Rhodoglobus as Rhodoglobus aureus comb. nov., and emended description of Rhodoglobus vestalii Sheridan et al. 2003 J Gen Appl Microbiol 56 53-5

Antibus D E, Leff L G, Hall B L, Baeseman J L and Blackwood C B (2012) Cultivable bacteria from ancient algal mats from the McMurdo Dry Valleys, Antarctica Extremophiles 16 $105-114$

Archer S D, McDonald I R, Herbold C W and Cary S C (2014) Characterisation of bacterioplankton communities in the meltwater ponds of Bratina Island, Victoria Land, Antarctica FEMS Microbiol Ecol 89 451-464

Bajerski F and Wagner D (2013) Bacterial succession in Antarctic soils of two glacier forefields on Larsemann Hills, East Antarctica FEMS Microbiol Ecol 85 128-142

Bottos E M, Woo A C, Zawar-Reza P, Pointing S B and Cary S C (2014) Airborne bacterial populations above desert soils of the McMurdo Dry Valleys, Antarctica Microb Ecol 67 $120-128$

Bowman J P, McCammon S A, Brown M V, Nichols D S and McMeekin T A (1997) Diversity and association of psychrophilic bacteria in Antarctic sea ice Appl Environ Microbiol 63 3068-3078

Bowman J P, Gosink J J, McCammon S A, Lewis T E, Nichols D S, Nichols P D, Skerratt J H, Staley J T and McMeekin T A (1998) Colwellia demingiae sp. nov., Colwellia hornerae sp. nov., Colwellia rossensis sp. nov. and Colwellia psychrotropica sp. nov.: psychrophilic Antarctic species with the ability to synthesize docosahexanoic acid (22:6 omega 3) Int J Syst Bacterial 48 1171-1180

Bowman J P, Rea S M, McCammon S A and McMeekin T A (2000) Diversity and community structure within anoxic sediment from marine salinity meromictic lakes and a coastal meromictic marine basin, Vestfold Hilds, Eastern Antarctica Environ Microbiol 2 227-237 
Boyd W L (1962) Comparison of soil bacteria and their metabolic activities in Arctic and Antarctic regions Polar Rec 11319

Boyd W L and Boyd J W (1962) Presence of Azotobacter species in polar regions $J$ Bacteriol 85 1121-1123

Bozal N, Montes M J and Mercadé E (2007) Pseudomonas guinea sp. nov., a novel psychrotolerant bacterium from an Antarctic environment Int J Syst Evol Microbiol 57 2609-2612

Brinkmeyer R, Knittel K, J“urgens J, Weyland H, Amann R and Helmke E (2003) Diversity and structure of bacterial communities in Arctic versus Antarctic pack ice Appl Environ Microbiol $696610-6619$

Brown M V and Bowman J P (2001) A molecular phylogenetic survey of sea-ice microbial communities (SIMCO) FEMS Microbiol Ecol 35 267-275

Campbell I B and Claridge G G C (2000) In: Antarctic Ecosystems: Models for Wider Understanding, W. Davidson, C. Howard-Williams and P. Broady (eds.)

Carpenter E J, Lin S and Capone D G (2000) Bacterial activity in South Pole snow Appl Environ Microbiol 66 4514-4517

Carr S A, Vogel S W, Dunbar R B, Brandes J, Spear J R, Levy R, Naish T R, Powell R D, Wakeham S G and Mandernack K W (2013) Bacterial abundance and composition in marine sediments beneath the Ross Ice Shelf, Antarctica Geobiology 11 377-395

Cavanagh J, Austin J J and Sanderson K (1996) Novel Psychrobacter species from Antarctic ornithogenic soils Int J Syst Bacterial 46 841-848

Cavicchioli R and Thomas T (2000) Extremophiles. In. Encyclopedia of Microbiology. Second Edition (J. Lederberg, Ed.), Vol 2, p 317-337, Academic Press, San Diego

Chaturvedi P, Prabahar V, Manorama R, Pindi P K, Bhadra B, Begum $\mathrm{Z}$ and Shivaji S (2008) Exiguobacterium soli sp. nov., a psychrophilic bacterium from the McMurdo Dry Valleys, Antarctica Int J Syst Evol Microbiol 58 2447-53

Chattopadhyay M K, Uma Devi K, Gopishankar Y and Shivaji S (1995) Thermolabile alkaline phosphatase from Sphingobacterium antarcticus, a psychrotrophic bacterium from Antarctica Polar Biol 15 215-219

Chattopadhyay M K, Jagannadham M V, Vairamani M and Shivaji S (1997) Carotenoid pigments of an antarctic psychrotrophic bacterium Micrococcus roseus: temperature dependent biosynthesis, structure, and interaction with synthetic membranes Biochem Biophys Res Commun 239 85-90

Chattopadhyay M K, Reddy, G S N and Shivaji S (2014)
Psychrophilic bacteria: Biodiversity, Molecular basis of cold adaptation and biotechnological implications Curr Biotech 3 100-116

Chessa J P, Petrescu I, Bentahir M, Van Beeumen J and Gerday C (2000) Purification, physico-chemical characterization and sequence of a heat labile alkaline metalloprotease isolated from a psychrophilic Pseudomonas species Biochim Biophys Acta 1479 265-274

Chintalapati S, Kiran M D and Shivaji S (2004) Role of membrane lipid fatty acids in cold adaptation Cell Mol Biol (Noisyle-grand) 50 631-42

Chintalapati S, Prakash J S, Gupta P, Ohtani S, Suzuki I, Sakamoto T, Murata N and Shivaji S (2006) A novel Delta9 acyllipid desaturase, DesC2, from cyanobacteria acts on fatty acids esterified to the sn-2 position of glycerolipids Biochem J 398 207-14

Chintalapati S, Prakash J S, Singh A K, Ohtani S, Suzuki I, Murata N and Shivaji S (2007) Desaturase genes in a psychrotolerant Nostoc sp. are constitutively expressed at low temperature Biochem Biophys Res Commun 362 81-7

Claridge G G C and Campbell I B (1977) The salts in Antarctic soils, their distribution and relationship to soil processes Soil Sci 123 377-384

Cristóbal H A, López M A, Kothe E and Abate C M (2011) Diversity of protease-producing marine bacteria from subantarctic environments J Basic Microbiol 51 590-600

D'Amico S, Claverie P, Collins T, Georlette D, Gratia E, Hoyoux A, Meuwis MA, Feller G and Gerday C (2002) Molecular basis of cold adaptation. Philos Trans R Soc Lond B Biol Sci 357 917-25

Davail S, Feller G, Narinx E and Gerday C (1994) Cold adaptation of proteins. Purification, characterization, and sequence of the heat-labile subtilisin from the antarctic psychrophile Bacillus TA41 J Biol Chem 269 $17448-53$

Delille D and Lagarde E (1974) Contribution a!'etude ecologique des Milieux subantarctiques $V$ Rev lnst Pasteur Lyon 7 149-165

Deming J W and Eicken H (2007) Life in ice. In: Baross, J. and W. Sullivan (eds.) Planets and life - The emerging science of astrobiology, Cambridge University Press, Cambridge, $p$ 292-312

De Domenico M, Lo Giudice A, Michaud L, Saitta M and Bruni V (2004) Diesel oil and PCB- degrading psychrotrophic bacteria isolated from Antarctic seawaters (Terra Nova Bay, Ross Sea) Polar Research 23 141-146

Delille D and Gleizon F (2003) Distribution of enteric bacteria in 
Antarctic seawater surrounding the Port-aux-Français permanent station (Kerguelen Island) Mar Pollut Bull 46 1179-1183

Denner E B M, Mark B, Busse H J, Turkiewicz M and Lubitz W (2001) Psychrobacter proteolyticus sp. nov., a psychrotrophic, halotolerant bacterium isolated from the Antarctic Krill Euphausia superba Dana, Excreting a coldadapted metalloprotease Syst Appl Microbiol 24 44-53

de Pascale D, Cusano A M, Autore F, Parrilli E, di Prisco G, Marino G and Tutino M L (2008) The cold-active Lip1 lipase from the Antarctic bacterium Pseudoalteromonas haloplanktis TAC125 is a member of a new bacterial lipolytic enzyme family Extremophiles 12 311-23

Doyle S M, Montross S N, Skidmore M L and Christner B C (2013) Characterizing microbial diversity and the potential for metabolic function at $-15^{\circ} \mathrm{C}$ in the Basal ice of taylor glacier, antarctica Biology (Basel) 2 1034-1053

Duilio A, Madonna S, Tutino M L, Pirozzi M, Sannia G and Marino G (2004) Promoters from a cold-adapted bacterium: definition of a consensus motif and molecular characterization of UP regulative elements Extremophiles $\mathbf{8}$ 125-32

Ekelof E (1908a) Bakteriologische studien wahrend der Schwedishen Sudpolar expedition 1901-1903. In: Wissenschaftiche Ergebnisse der Schwedischen sudpolar Expedition 1901-1903. 0. Nordenskjold (ed.) Lithogr. lnst.Generalstabs, Stockholm.210

Ekelof E (1908b) Studien uber den Bakteriengehalt der luft und des Erdbodeus der antarktishen Gegenden, ausgefiihrt wahrend der schwedischen sudpolar expedition 1901-1903. ZHyg lnfekt 56 344-370

Feller G, Zekhnini Z, Lamotte-Brasseur J and Gerday C (1997) Enzymes from cold-adapted microorganisms. The class $\mathrm{C}$ beta-lactamase from the antarctic psychrophile Psychrobacter immobilis A5 Eur J Biochem 244 186-91

Finegold L (1986) Molecular aspects of adaptation to extreme cold environments Adv Space Res 6 257-64

Frühling A, Schumann P, Hippe H, Sträubler B and Stackebrandt E (2002) Exiguobacterium undae sp. nov. and Exiguobacterium antarcticum sp. nov Int J Syst Evol Microbiol 52 1171-6

Franzmann P D, Deprez P P, MeGuire A J, McMeekin T A and Burton H R (1990) The Heterotrophic, Bacterial Microbiota of Burton Lake, Antarctica Polar Biol 10261 264

Franzmann P D, Höpfl P, Weiss N and Tindall B J (1991) Psychrotrophic, lactic acid-producing bacteria from anoxic waters in Ace Lake, Antarctica; Carnobacterium funditum sp. nov. and Carnobacterium alterfunditum sp. nov Arch Microbiol 156 255-62

Franzmann P D and Dobson S J (1992) Cell wall-less, free-living spirochetes in Antarctica FEMS Microbiol Lett 76 289292

Friedman E 1 (1980) Endolithic microbial life in hot and cold deserts Orig Life 10 223-235

Ganzert L, Bajerski F, Mangelsdorf K, Lipski A and Wagner D (2011) Arthrobacter livingstonensis sp. nov. and Arthrobacter cryotolerans sp. nov., salt-tolerant and psychrotolerant species from Antarctic soil Int J Syst Evol Microbiol 61 979-84

Gazert H (1912) Undersuchungen uber Meeresbakterien und chren Einfluss auf den Stoffwecksel im Meer. Deutschi biidpolar expedition. 1901-1903 Berlin, 7 268-296

Georlette D, Jónsson Z O, Van Petegem F, Chessa J, Van Beeumen J, Hübscher U and Gerday C (2000) A DNA ligase from the psychrophile Pseudoalteromonas haloplanktis gives insights into the adaptation of proteins to low temperatures Eur J Biochem 267 3502-12

Gerday C, Aittaleb M, Bentahir M, Chessa J P, Claverie P, Collins T, D'Amico S, Dumont J, Garsoux G, Georlette D, Hoyoux A, Lonhienne T, Meuwis M A and Feller G (2000) Coldadapted enzymes: from fundamentals to biotechnology Trends Biotechnol 18 103-7

Gosink J J, Woese C R and Staley J T (1998) Polaribacter gen. nov., with three new species, $P$. irgensii sp. nov., $P$. franzmannii sp. nov., and $P$. filamentus sp. nov., gas vacuolate polar marine bacteria of the CytophagaFlavobacterium-Bacteroides group and reclassification of Flectobacillus glomeratus as Polaribacter glomeratus comb. nov Int J Syst Bacteriol 48 223-235

Guibert L M, Loviso C L, Marcos M S, Commendatore M G, Dionisi H M and Lozada M (2012) Alkane biodegradation genes from chronically polluted subantarctic coastal sediments and their shifts in response to oil exposure Microb Ecol 64 605-616

Helmke E and Weyland H (2004) Psychrophilic versus psychrotolerant bacteria-occurrence and significance in polar and temperate marine habitats Cell Mol Biol 50 553561

Herbert R A and Bell C R (1977) Growth characteristics of an obligately psychrophilic Vibrio sp Arch Microbiol 113 215-20

Hirsch P, Mevs U, Kroppenstedt R M, Schumann P and Stackebrandt E (2004) Cryptoendolithic actinomycetes from antarctic sandstone rock samples: Micromonospora endolithica sp. nov. and two isolates related to 
Micromonospora coerulea Jensen 1932 Syst Appl Microbiol 27 166-174

Hong S G, Lee Y K, Yim J H, Chun J and Lee H K (2008) Sanguibacter antarcticus sp. nov., isolated from Antarctic sea sand Int J Syst Evol Microbiol 58 50-2

Hoyoux A, Jennes I, Dubois P, Genicot S, Dubail F, François J M, Baise E, Feller G and Gerday C (2001) Cold-adapted beta-galactosidase from the Antarctic psychrophile Pseudoalteromonas haloplanktis Appl Environ Microbiol 67 1529-35

Huang Y, Zhang J and Zhu L (2013) Evaluation of the application potential of bentonites in phenanthrene bioremediation by characterizing the biofilm community Bioresour Technol 134 17-23

Imperio T, Viti C and Marri L (2008) Alicyclobacillus pohliae sp. nov., a thermophilic, endospore-forming bacterium isolated from geothermal soil of the north-west slope of Mount Melbourne (Antarctica) Int J Syst Evol Microbiol 58221 225

Irgens R L, Gosink J J and Staley J T (1996) Polaromonas vacuolata gen. nov., sp. nov., a psychrophilic, marine, gas vacuolate bacterium fromAntarctica Int J Syst Bacteriol $\mathbf{4 6}$ 822-6

Jagannadham M V, Rao V J and Shivaji S (1991) The major carotenoid pigment of a psychrotrophic Micrococcus roseus strain: purification, structure, and interaction with synthetic membranes J Bacteriol 173 7911-7

Jagannadham M V, Chattopadhyay M K and Shivaji S (1996) The major carotenoid pigment of a psychrotrophic Micrococcus roseus strain: fluorescence properties of the pigment and its binding to membranes Biochem Biophys Res Commun 220 724-8

Jagannadham M V, Chattopadhyay M K, Subbalakshmi C, Vairamani M, Narayanan K, Rao C M and Shivaji S (2000) Carotenoids of an Antarctic psychrotolerant bacterium, Sphingobacterium antarcticus, and a mesophilic bacterium, Sphingobacterium multivorum. Arch Microbiol 173 418-24

Janiyani K L and Ray M K (2002) Cloning, sequencing, and expression of the cold-inducible hutU gene from the antarctic psychrotrophic bacterium Pseudomonas syringae Appl Environ Microbiol 68 1-10

Jovcic B, Bertani I, Venturi V, Topisirovic L and Kojic M (2008) 5' Untranslated region of the Pseudomonas putida WCS358 stationary phase sigma factor rpoSmRNA is involved in RpoS translational regulation J Microbiol 46 56-61

Junge K, Imhoff F, Staley T and Deming J W (2002) Phylogenetic diversity of numerically important Arctic sea-ice bacteria cultured at subzero temperature Microb Ecol 43 315-328

Junge K, Eicken H and Deming J W (2004) Bacterial activity at 2 to $-20^{\circ} \mathrm{C}$ in Arctic wintertime sea ice Appl Environ Microbiol 70 550-557

Karl D M, Bird D F, Björkman K, Houlihan T, Shackelford R and Tupas L (1999) Microorganisms in the accreted ice of Lake Vostok, Antarctica Science 286 2144-2147

Karr E A, Sattley W M, Jung D O, Madigan M T and Achenbach L A (2003) Remarkable diversity of phototrophic purple bacteria in a permanently frozen Antarctic lake Appl Environ Microbiol 69 4910-4914

Karr E A, Sattley W M, Rice M R, Jung D O, Madigan M T and Achenbach LA (2005) Diversity and distribution of sulfatereducing bacteria in permanently frozen Lake Fryxell, McMurdo Dry Valleys, Antarctica Appl Environ Microbiol 71 6353-6359

Kelly M D, Lukaschewsky S and Anderson C G (1978) Bacterial flora of Antarctic krill and some of their enzymatic properties J Food Sci 43 1196-1197

Kim J G, Park S J, Quan Z X, Jung M Y, Cha I T, Kim S J, Kim K H, Yang E J, Kim Y N, Lee S H and Rhee S K (2014) Unveiling abundance and distribution of planktonic Bacteria and Archaea in a polynya in Amundsen Sea, Antarctica Environ Microbiol 16 566-578

Kimes N E, Callaghan A V, Suflita J M and Morris P J (2014) Microbial transformation of the Deepwater Horizon oil spill-past, present, and future perspectives Front Microbiol 5603

Kiran M D, Annapoorni S, Suzuki I, Murata N and Shivaji S (2005) Cis-trans isomerase gene in psychrophilic Pseudomonas syringae is constitutively expressed during growth and under conditions of temperature and solvent stress Extremophiles 9 117-25

Kong W, Li W, Romancova I, Prášil O and Morgan-Kiss R M (2014) An integrated study of photochemical function and expression of a key photochemical gene (psbA) in photosynthetic communities of Lake Bonney (McMurdo Dry Valleys, Antarctica) FEMS Microbiol Ecol 89 293302

Kuhn E, Bellicanta G S and Pellizari V H (2009) New alk genes detected in Antarctic marine sediments Environ Microbiol 11 669-673

Kuhn E, Ichimura A S, Peng V, Fritsen C H, Trubl G, Doran P T and Murray A E (2014) Brine assemblages of ultra small microbial cells within the ice cover of Lake Vida, Antarctica Appl Environ Microbiol 80 3687-3698 
Kumar P A, Sreenivas A, Aditya Singh and Shivaji S (2013a) Draft genome sequence of Winogradskyella psychrotolerans RS-3T, isolated from the marine transect of Kongsfjorden, Ny-Ålesund, Svalbard, Arctic Genome Announc 1 e0063013

Kumar P A, Aditya Singh, Sreenivas A, Begum Z, Reddy G S N and Shivaji S (2013b) Draft genome sequence of Leifsoniarubra CMS 76R T, isolated from a cyanobacterial mat sample from a pond in Wright Valley, McMurdo, Antarctica Genome Announc 1 e00633-13

Labrenz M, Collins M D, Lawson P A, Tindall B J, Schumann P and Hirsch P (1999) Roseovarius tolerans gen. nov., sp. nov., a budding bacterium with variable bacteriochlorophyll a production from hypersaline Ekho Lake Int J Syst Bacteriol 49 137-47

Labrenz M, Tindall B J, Lawson P A, Collins M D, Schumann P and Hirsch P (2000) Staleya guttiformis gen. nov., sp. nov. and Sulfitobacter brevis sp. nov., alpha-3-Proteobacteria from hypersaline, heliothermal and meromictic Antarctic Ekho Lake Int J Syst Evol Microbial 50 303-313

Labrenz M, Lawson P A, Tindall B J, Collins M D and Hirsch P (2003) Saccharospirillum impatiens gen. nov., sp. nov., a novel gamma-Proteobacterium isolated from hypersaline Ekho Lake (East Antarctica) Int J Syst Evol Microbiol 53 653-60

Labrenz M, Lawson P A, Tindall B J, Collins M D and Hirsch P (2005) Roseisalinus antarcticus gen. nov., sp. nov., a novel aerobic bacteriochlorophyll a-producing alphaproteobacterium isolated from hypersaline Ekho Lake, Antarctica Int J Syst Evol Microbiol 55 41-7

Labrenz M, Lawson P A, Tindall B J and Hirsch P (2009) Roseibaca ekhonensis gen. nov., sp. nov., an alkalitolerant and aerobic bacteriochlorophyll a-producing alphaproteobacterium from hypersaline Ekho Lake Int $J$ Syst Evol Microbiol 59 1935-40

Lama L, Nicolaus B, Calandrelli V, Esposito E and Gambacorta A (1996) Xylanase produced by Bacillus thermoantarcticus, a new thermophilic bacillus Enzyme Eng XIII 284-286

Lanoil B, Skidmore M, Priscu J C, Han S, Foo W, Vogel S W, Tulaczyk S and Engelhardt H (2009) Bacteria beneath the West Antarctic ice sheet Environ Microbiol 11 609-615

Larkin J M and Stokes J L (1967) Taxonomy of psychrophilic strains of Bacillus J Bacteriol 94 889-895

Laucks M L, Sengupta A, Junge K, Davis E J and Swan-son B D (2005) Comparison of psychro-active Arctic marine bacteria and common mesophilic bacteria using surfaceenhanced Raman spectroscopy Appl Spectr 10 1222-1228

Lauro F M, Chastain R A, Blankenship L E, Yayanos A A and
Bartlett D H (2007) The unique 16S rRNA genes of piezophiles reflect both phylogeny and adaptation Appl Environ Microbiol 73 838-845

Lee K, Lee H K, Choi T H, Kim K M and Cho J C (2007) Granulosicoccaceae fam. nov., to include Granulosicoccus antarcticus gen. nov., sp. nov., a non-phototrophic, obligately aerobic chemoheterotroph in the order Chromatiales, isolated from Antarctic seawater J Microbiol Biotechnol 17 1483-1490

Lee C K, Barbier B A, Bottos E M, McDonald I R and Cary S C (2012) The Inter-Valley Soil Comparative Survey: the ecology of Dry Valley edaphic microbial communities ISME J 6 1046-1057

Lee L H, Cheah Y K, Sidik S M, Xie Q Y, Tang Y L, Lin H P, Mutalib N S and Hong K (2013) Barrientosiimonas humi gen. nov., sp. nov., an actinobacterium of the family Dermacoccaceae Int J Syst Evol Microbiol 63 241-8

Li H R, Yu Y, Luo W and Zeng Y X (2010) Marisediminicola antarctica gen. nov., sp. nov., an actinobacterium isolated from the Antarctic Int J Syst Evol Microbiol $602535-9$

Li S, Xiao X, Yin X and Wang F (2006) Bacterial community along a historic lake sediment core of Ardley island west Antarctica Extremophile 10 461-467

Li J, Tian X P, Zhu T J, Yang L L and Li W J (2011) Streptomyces fildesensis sp. nov., a novel streptomycete isolated from Antarctic soil.Antonie Van Leeuwenhoek 100 $537-43$

Liu C, Zhang X Y, Su H N, Zhou M Y, Chen B, Li H, Chen X L, Zhao D L, Zhou B C, Shi M, Zhang Y Z (2014) Puniceibacterium antarcticum gen. nov., sp. nov., isolated from seawater Int J Syst Evol Microbiol 64 1566-72

Logan N A, Lebbe L, Hoste B, Goris J, Forsyth G, Heyndrickx M, Murray B L, Syme N, Wynn-Williams D D and De Vos P (2000) Aerobic endospore-forming bacteria from geothermal environments in northern Victoria Land,Antarctica, and Candlemas Island, South Sandwich archipelago, with the proposal of Bacillus fumarioli sp. nov Int J Syst Evol Microbiol 50 1741-53

Logan N A, De Clerck E, Lebbe L, Verhelst A, Goris J, Forsyth G, Rodríguez-Díaz M, Heyndrickx M and De Vos P (2004) Paenibacillus cineris sp. nov. and Paenibacillus cookii sp. nov., from Antarctic volcanic soils and a gelatin-processing plant Int J Syst Evol Microbiol 54 1071-6

Lo Giudice A, Caruso C, Mangano S, Bruni V, De Domenico M and Michaud L (2012) Marine bacterioplankton diversity and community composition in an antarctic coastal environment Microb Ecol 63 210-223

Maas E W, Simpson A M, Martin A, Thompson S, Koh E Y, 
Davy S K, Ryan K G and O'Toole R F (2012) Phylogenetic analyses of bacteria in sea ice at Cape Hallett, Antarctica $N$ Z J Mar Freshw Res 46 3-12

Madigan M T, Jung D O, Woese C R and Achenbach L A (2000) Rhodoferax antarcticus sp. nov., a moderately psychrophilic purple nonsulfur bacterium isolated from an Antarctic microbial mat Arch Microbiol 173 269-77

Magalhães C M, Machado A, Frank-Fahle B, Lee C K and Cary S C (2014) The ecological dichotomy of ammonia-oxidizing archaea and bacteria in the hyper-arid soils of the Antarctic Dry Valleys Front Microbiol 5515

Marcos M S, Lozada M and Dionisi H M (2009) Aromatic hydrocarbon degradation genes from chronically polluted Subantarctic marine sediments Lett Appl Microbiol 49 602608

Margesin R and Schinner F (2001) Biodegradation and bioremediation of hydrocarbons in extreme environments Appl Microbiol Biotechnol 56 650-663

Margini R A and Castrelos O D (1963) Examenes bacteriologicos de aire, rieve y suelo de carbo primaruera y Estacion cientifica Ellsworth Inst Antarcti Argent Publ 76 1-15

Margini R A and Castrelos O D (1965) Las bacterias del Antarctico y su relacion con la epoca del ano contrib Inst Antarct Argent 141 1-19

Marshall B J and Ohye D F (1966) Bacillus macquariensis sp. nov, a psychrotrophic bacterium from sub-antarctic soil $J$ Gen Microbiol 44 41-6

Master E R and Mohn W W (1998) Psychrotolerant bacteria Isolated from Arctic Soil that degrade polychlorinated biphenyls at low temperatures Appl Environ Microbiol 64 4823-4829

McLean A L (1918a) Bacteria of ice and snow in Antarctica Nature 102 35-39

McLean A L (1918b) Bacteriological and other researches Australian Antarctic Expedition 1911-1914 Scient Rep 7 $1-128$

Metpally R P and Reddy B V (2009) Comparative proteome analysis of psychrophilic versus mesophilic bacterial species: Insights into the molecular basis of cold adaptation of proteins BMC Genomics $\mathbf{1 0} 11$

Mevs U, Stackebrandt E, Schumann P, Gallikowski C A and Hirsch P (2000) Modestobacter multiseptatus gen. nov., sp. nov., a budding actinomycete from soils of the Asgard Range (Transantarctic Mountains) Int J Syst Evol Microbiol $50337-46$

Meyer G H, Morrow M B, Wyss O, Berg T E and Littlepage J L (1962) Antarctica: The microbiology of an unfrozen saline pond Science 138 1103-4

Michaud L, Caruso C, Mangano S, Interdonato F, Bruni V and Lo Giudice A (2012) Predominance of Flavobacterium, Pseudomonas, and Polaromonas within the prokaryotic community of freshwater shallow lakes in the northern Victoria Land, East Antarctica FEMS Microbiol Ecol 82 391-404

Miwa T (1975) Clostridia in soil of the Antarctica Jpn J Med Sci Biol 28 201-213

Moreno R and Rojo F (2014) Features of pseudomonads growing at low temperatures: another facet of their versatility Environ Microbiol Rep 6 417-426

Morita RY (1975) Psychrophilic Bacteria Bacteriol Rev 39 144167

Muangchinda C, Chavanich S, Viyakarn V, Watanabe K, Imura S, Vangnai A S and Pinyakong O (2014) Abundance and diversity of functional genes involved in the degradation of aromatic hydrocarbons in Antarctic soils and sediments around Syowa Station Environ Sci Pollut Res 22 47254735

Niederberger T D, McDonald I R, Hacker A L, Soo R M, Barrett J E, Wall D H and Cary S C (2008) Microbial community composition in soils of Northern Victoria Land, Antarctica Environ Microbiol 10 1713-1724

Pavankumar T L, Sinha A K and Ray M K (2010) All three subunits of RecBCD enzyme are essential for DNA repair and low-temperature growth in the Antarctic Pseudomonas syringae Lz4W PLoS One 5 e9412

Peeters K, Hodgson D A, Convey P and Willems A (2011) Culturable diversity of heterotrophic bacteria in Forlidas Pond (Pensacola Mountains) and Lundström Lake (Shackleton Range), Antarctica Microb Ecol 62 399-413

Pfiser R M and Burkholder P R (1965) Numerical taxonomy of some bacteria isolated from Antarctic and tropical sea waters J Bacterial 90 863-872

Pirie J H H (1904) First Antarctic voyage of the Scotia J Bacterial Scottish Geog Mag 20 129-132

Poli A, Esposito E, Lama L, Orlando P, Nicolaus G, de Appolonia F, Gambacorta A and Nicolaus B (2006) Anoxybacillus amylolyticus sp. nov., a thermophilic amylase producing bacterium isolated from Mount Rittmann (Antarctica) Syst Appl Microbiol 29 300-307

Powell S M, Ferguson S H, Bowman J P and Snape I (2006) Using real-time PCR to assess changes in the hydrocarbondegrading microbial community in Antarctic soil during bioremediation Microb Ecol 52 523-532

Prabagaran S R, Manorama R, Delille D and Shivaji S (2007) 
Predominance of Roseobacter, Sulfitobacter, Glaciecola and Psychrobacter in seawater collected off Ushuaia, Argentina, Sub-Antarctica FEMS Microbiol Ecol 59 342-355

Priest F G, Goodfellow M and Todd C (1988) A numerical classification of the genus Bacillus J Gen Microbiol 134 1847-1882

Priscu J C, Adams E E, Lyons W B, Voytek M A, Mogk D W, Brown R L, McKay C P, Takacs C D, Welch K A, Wolf C F, Kirshtein J D and Avci R (1999) Geomicrobiology of subglacial ice above Lake Vostok, Antarctica Science $\mathbf{2 8 6}$ 2141-2144

Prüss B M, Francis K P, von Stetten F and Scherer S (1999) Correlation of $16 \mathrm{~S}$ ribosomal DNA signature sequences with temperature-dependent growth rates of mesophilic and psychrotolerant strains of the Bacillus cereus Group J Bacteriol 181 2624-2630

Purcell A M, Mikucki J A, Achberger A M, Alekhina I A, Barbante C, Christner B C, Ghosh D, Michaud A B, Mitchell A C, Priscu J C, Scherer R, Skidmore M L and Vick-Majors T J, The Wissard Science Team (2014) Microbial sulfur transformations in sediments from Subglacial Lake Whillans Front Microbiol 5594

Ramsay A J and Stannard R E (1986) Numbers and viability of bacteria in ornithogenic soils of Antarctica Polar Biol 5 195-198

Ray M K, Devi K U, Kumar G S and Shivaji S (1992) Extracellular protease from the antarctic yeast Candida humicola Appl Environ Microbiol 58 1918-23

Ray M K, Kumar G S and Shivaji S (1994a) Phosphorylation of lipopolysaccharides in the Antarctic psychrotroph Pseudomonas syringae: a possible role in temperature adaptation J Bacteriol 176 4243-9

Ray M K, Kumar G S and Shivaji S (1994b) Phosphorylation of lipopolysaccharides in the Antarctic psychrotroph Pseudomonas syringae: a possible role in temperature adaptation J Bacteriol 176 4243-9

Ray M K, Kumar G S, Janiyani K, Kannan K, Jagtap P, Basu M K and Shivaji S (1998) Adaptation to low temperature and regulation of gene expression in Antarctic psychrotrophic bacteria J Biosci 23 423-435

Reddy G S N, Rajagopalan G and Shivaji S (1994) Thermolabile ribonuclease from Antarctic psychrotropic bacteria: detection of the enzyme in various bacteria and purification from Pseudomonas fluorescens FEMS Microbiol Lett 122 211-216

Reddy G S N, Agarwal R K, Matsumoto G I and Shivaji S (2000) Arthrobacter flavus sp. nov., a psychrotropic bacterium isolated from a pond in Mc Murdo Dry Valley, Antarctica
Int J Syst Evol Microbiol 50 1553-1561

Reddy G S N, Prakash J S, Matsumoto G I, Stackebrandt E and Shivaji S (2002) Arthrobacter_roseus sp. nov., a psychrophilic bacterium isolated from an antarctic cyanobacterial mat sample. Int J Syst Evol Microbiol 52 1017-21

Reddy G S N, Matsumoto G I and Shivaji S (2003a) Sporosarcina macmurdoensis sp. nov. from a cyanobacterial mat samples from a pond in the McMurdo dry valley, Antarctica Int J Syst Evol Microbiol 53 1363-1367

Reddy G S N, Prakash J S S, Srinivas R, Matsumoto G I and Shivaji S (2003b) Leifsonia rubra sp. nov. and Leifsonia aurea sp. nov. psychrophiles from a Pond in Antarctica Int J Syst Evol Microbiol 53 977-984

Reddy G S N, Matsumoto G I, Shuman P, Stackebrandt E and Shivaji S (2004) Psychrophilic Pseudomonas from Antarctica: Pseudomonasantarctica sp. nov., Pseudomonas meridianae sp. nov. and Pseudomonas proteolytica sp. nov Int J Syst Evol Microbiol 54 713-719

Reddy G S N, Uttam A and Shivaji S (2008) Bacillus cecembensis sp. nov., a bacterium isolated from the Pindari glacier of the Himalayan mountain ranges, India Int J Syst Evol Microbiol 58 2330-2335

Reddy G S N, Ara S, Singh A, Kumar Pinnaka A and Shivaji S (2013) Draft genome sequence of Psychrobacter aquaticus CMS 56T, isolated from a cyanobacterial mat sample collected from water bodies in the McMurdo Dry Valley region of Antarctica Genome Announc 1 e00918-13

Reddy G S N, Sreenivas A and Shivaji S (2014) Draft genome sequence Cryobacterium roopkundensis strain RuG17, isolated from a soil sample in the vicinity of Roopkund Lake, Himalayas, India Genome Announc 2 e01206-14

Reddy G S N, Sreenivas A and Shivaji S (2015) Draft genome of Kocuria polaris CMS 76or ${ }^{\mathrm{T}}$ isolated from cyanobacterial mats, McMurdo Dry Valley, Antarctica: an insight into CspA family of proteins from Kocuria polaris CMS 76or ${ }^{\mathrm{T}}$ Arch Microbiol 197 1019-1026

Reddy G S N, Chattopadhyay M K and Shivaji S (2016) Biodiversity, Adaptation and Biotechnological importance of bacteria occurring in cold climates. In. Biotechnology of Extremophiles: Advances and Challenges. Ed. Pabulo H. Rampelotto (in press).

Rina M, Pozidis C, Mavromatis K, Tzanodaskalaki M, Kokkinidis M and Bouriotis V (2000) Alkaline phosphatase from the Antarctic strain TAB5. Properties and psychrophilic adaptations Eur J Biochem 267 1230-8

Ruckert G (1985) Myxobacteria from Antarctic soils Biol Fert Soil 1 215-216 
Russell R J, Gerike U, Danson M J, Hough D W and Taylor G L (1998) Structural adaptations of the cold-active citrate synthase from an Antarctic bacterium Structure 6 351-61

Sheridan P P, Loveland-Curtze J, Miteva V I and Brenchley J E (2003) Rhodoglobus vestalii gen. nov., sp. nov., a novel psychrophilic organism isolated from an Antarctic Dry Valley lake Int J Syst Evol Microbiol 53 985-94

Shivaji S, Rao N S, Saisree L, Sheth V, Reddy G S N and Bhargava P M (1988) Isolation and identification of Micrococcus roseus and Planococcus sp. from Schirmacher Oasis, Antarctica J Biosci 113 409-414

Shivaji S, Rao N S, Saisree L, Sheth V, Reddy G S N and Bhargava P M (1989a) Isolation and identification of Pseudomonas sp. from Schirmacher Oasis, Antarctica Appl Environ Microbiol 55 767-770

Shivaji S, Rao N S, Saisree L, Reddy G S N, Seshu Kumar G and Bhargava P M (1989b) Isolates of Arthrobacter from the soils of Schirmacher Oasis, Antarctica Polar Biol 10225 229

Shivaji S, Ray M K, Kumar G S, Reddy G S N, Saisree L and Wynn-Williams D D (1991) Identification of Janthinobacterium lividum from the soils of the Island of Scotia Ridge from Antarctic Peninsula Polar Biol $11267-$ 272

Shivaji S Reddy G S N, Prasad R A, Kutty R and Ravenschlag K (2004) Bacterial diversity of a soil sample from Schirmacher Oasis, Antarctica Cell Mol Biol 50 525-536

Shivaji S, Kiran M D and Chintalapati S (2007) Perception and transduction of low temperature in bacteria. In: Physiology and biochemistry of extremophiles. Gerday C, Glansdor VN (Ed) ASM Press, Washington, 194-207

Shivaji S and Reddy G S N (2009) Chapter 2. Bacterial biodiversity of Antarctica: conventional polyphasic and rRNA approaches. In: Polar Microbiology: The Ecology, Biodiversity and Bioremediation Potential of Microorganisms in Extremely Cold Environments; Bej A K, Aislabie J, Atlas R M, Eds. Taylor \& Francis group

Shivaji S and Prakash J S (2010) How do bacteria sense and respond to low temperature? Arch Microbiol 192 85-95

Shivaji S, Kumari K, Kishore K H, Pindi P K, Rao P S, Radha Srinivas T N, Asthana R and Ravindra R (2011) Vertical distribution of bacteria in a lake sediment from Antarctica by culture-independent and culture-dependent approaches Res Microbiol 162 191-203

Shivaji S, Ara S, Singh S K, Bandi S, Singh A and Pinnaka A K (2012) Draft genome sequence of Bacillus Isronensis strain B3W22, isolated from the upper atmosphere $J$ Bacteriol 194 6624-6625
Shivaji S, Ara S, Singh A and Pinnaka A K (2013a) Draft genome sequence of Cyclobacterium qasimii M12-11B T, isolated from an Arctic marine sediment Genome Announc 1 e0064213

Shivaji S, Ara S, Prasad S, Manasa B P, Begum Z, Singh A and Pinnaka A K (2013b) Draft genome sequence of Arcticibacter svalbardensis MN12-7T, a member of the family Sphingobacteriaceae isolated from an Arctic soil sample Genome Announc 1 e00484-13

Shukor M Y, Hassan N A, Jusoh A Z, Perumal N, Shamaan N A, MacCormack W P and Syed M A (2009) Isolation and characterization of a Pseudomonas diesel-degrading strain from Antarctica $J$ Environ Biol 30 1-6

Singh A K, Pindi P K, Dube S, Sundareswaran V R and Shivaji S (2009) In the psychrophilic Pseudomonas syringae, trmE is important for low temperature growth Appl Environ Microbiol 75 4419-4426

Singh P, Singh SM and Dhakephalkar P (2014) Diversity, cold active enzymes and adaptation strategies of bacteria inhabiting glacier cryoconite holes of High Arctic Extremophiles 18 229-242

Sjöling S and Cowan D A (2003) High 16S rDNA bacterial diversity in glacial meltwater lake sediment, Bratina Island, Antarctica Extremophiles 7 275-282

Smith R C, Prezelin B B, Baker K S, Bidigare R R, Boucher N P, Coley T, Karentz D, MacIntyre S, Matlick H A, Menzies D, Ondrusek M, Wan Z and Waters K J (1992) Ozone depletion: ultraviolet radiation and phytoplankton biology in Antarctic waters Science 255 952-959

Søgaard D H, Kristensen M, Rysgaard S, Glud R N, Hansen P J and Hilligsøe K M (2010) Autotrophic and heterotrophic activity in Arctic first-year sea ice: Seasonal study from Malene Bight, SW Greenland Mar Ecol Prog Ser 419 3145

Söller R, Hirsch P, Blohm D and Labrenz M (2000) Differentiation of newly described antarctic bacterial isolates related to Roseobacter species based on 16S-23S rDNA internal transcribed spacer sequences Int J Syst Evol Microbiol 50 909-915

Spring S, Merkhoffer B, Weiss N, Kroppenstedt R M, Hippe H and Stackebrandt E (2003) Characterization of novel psychrophilic clostridia from an Antarctic microbial mat: description of Clostridium frigoris sp. nov., Clostridium lacusfryxellense sp. nov., Clostridium bowmanii sp. nov., and Clostridium psychrophilum sp. nov., and reclassification of Clostridium laramiense as Clostridium estertheticum subsp. laramiense subsp. nov Int J Syst Evol Microbiol 53 1019-1029 
Sreenivas A, Reddy G SN and Shivaji S (2014) Draft genome sequence of a psychrophilic bacterium Sphingomonas antarcticum 4BY, isolated from the soils of Schirmacher oasis, Antarctica Genome Announc 2 e00696-14

Stibal M, Hasan F, Wadham J L, Sharp M J and Anesio A M (2012) Prokaryotic diversity in sediments beneath two polar glaciers with contrasting organic carbon substrates Extremophiles 16 255-265

Stokes J L and Reymond M L (1966) Quantitative ecology of psychrophilic microorganisms Appl Microbiol 14 74-78

Sundareswaran V R, Singh A K, Dube S and Shivaji S (2010) Aspartate aminotransferase is involved in cold adaptation in psychrophilic Pseudomonas syringae Arch Microbiol 192 663-672

Takii S, Kondal T, Hiraishi A, Matsumoto G I, Kawano T and Torii T (1986) Vertical distribution in and isolation of bacteria from Lake Vanda: an Antarctic lake Hydrobiologia $13515-21$

Tanner A C and Herbert R A (1981) Nutrient regeneration in Antarctic marine sediments Kiel Meeresforsch Sanderh 5 390-395

Tanner A C (1985) The role of bacteria in the cycling of nutrients within the maritime Antarctic environment. Antarctic Nutrient Cycles and Food Webs (ed. W R Siegfried, P R Condy and Laws R M), Springer Verlag, Berlin, Heidelberg, p 123-127

Tiao G, Lee C K, McDonald I R, Cowan D A and Cary S C (2012) Rapid microbial response to the presence of an ancient relic in the Antarctic Dry Valleys Nat Commun 3660

Timmis K N, McGenity T J, Meer J R and deLorenzo V (eds.) (2010) Handbook of hydrocarbon and lipid microbiology. Berlin; Heidelberg: Springer Verlag. doi: 10.1007/978-3540-77587-4

Tsiklinsky M (1908) La flore microbieene dans les regions due pole Sud. In: Expedition Antarctique Francais 1903-1905. $31-33$

Tsyganov V A (1970) Detection and morphological cultural characteristics of Actinomycetes from the antarctic Mikrobiologia 39 821-826

Uma S, Jadhav R S, Kumar G S, Shivaji S and Ray M K (1999) A RNA polymerase with transcriptional activity at 0 degrees C from the Antarctic bacterium Pseudomonas syringae FEBS Lett 453 313-7

Van Trappen S, Mergaert J, Van Eygen S, Dawyndt P, Cnockaert M C and Swings J (2002) Diversity of 746 heterotrophic bacteria isolated from microbial mats from ten Antarctic lakes Syst Appl Microbiol 25 603-610
Van Trappen S, Tan T L, Yang J, Mergaert J and Swings J (2004) Glaciecola polaris sp. nov., a novel budding and prosthecate bacterium from the Arctic Ocean, and emended description of the genus Glaciecola Int J Syst Evol Microbiol 54 17651771

Vincent C F (1988) Microbial ecosystems of Antarctica. Cambridge: Cambridge University Press 303

Voytek M A and Ward B B (1995) Detection of ammoniumoxidizing bacteria of the beta-subclass of the class Proteobacteria in aquatic samples with the PCR Appl Environ Microbiol 61 1444-1450

Wang F, Gai Y, Chen M and Xiao X (2009) Arthrobacter psychrochitiniphilus sp. nov., a psychrotrophic bacterium isolated from Antarctica Int J Syst Evol Microbiol 592759 62

Watanabe T, Kojima H, Takano Y and Fukui M (2013) Diversity of sulfur-cycle prokaryotes in freshwater lake sediments investigated using aprA as the functional marker gene Syst Appl Microbiol 36 436-443

Wery N, Gerike U, Sharman A, Chaudhuri J B, Hough D W and Danson M J (2003) Use of a packed-column bioreactor for isolation of diverse protease-producing bacteria from antarctic soil Appl Environ Microbiol 69 1457-1464

Wilkins D, Yau S, Williams T J, Allen MA, Brown M V, DeMaere M Z, Lauro F M and Cavicchioli R (2013) Key microbial drivers in Antarctic aquatic environments FEMS Microbiol Rev 37 303-335

Xiao X, Yin X, Lin J, Sun L, You Z, Wang P and Wang F (2005) Chitinase genes in lake sediments of Ardley Island, Antarctica Appl Environ Microbiol 71 7904-7909

Xu S, Yan L, Zhang X, Wang C, Feng G and Li J (2014) Nocardiopsis fildesensis sp. nov., an actinomycete isolated from soil Int J Syst Evol Microbiol 64 174-9

Yakimov M M, Giuliano L, Gentile G, Crisafi E, Chernikova T N, Abraham W R, Lünsdorf H, Timmis K N and Golyshin P N (2003) Oleispira antarctica gen. nov., sp. nov., a novel hydrocarbonoclastic marine bacterium isolated fromAntarctic coastal sea water Int J Syst Evol Microbiol $\mathbf{5 3} 779-85$

Yamada K, Fukuda W, Kondo Y, Miyoshi Y, Atomi H and Imanaka T (2011) Constrictibacter antarcticus gen. nov., sp. nov., a cryptoendolithic micro-organism from Antarcticwhite rock Int J Syst Evol Microbiol 61 1973-80

Yau S, Lauro F M, Williams T J, Demaere M Z, Brown M V, Rich J, Gibson J A and Cavicchioli R (2013) Metagenomic insights into strategies of carbon conservation and unusual sulfur biogeochemistry in a hypersaline Antarctic lake Int J Syst Evol Microbiol 7 1944-1961 
Yergeau E, Newsham K K, Pearce D A and Kowalchuk G A (2007a) Patterns of bacterial diversity across a range of Antarctic terrestrial habitats Environ Microbiol 9 26702682

Yergeau E, Bokhorst S, Huiskes A H, Boschker H T, Aerts R and Kowalchuk G A (2007b) Size and structure of bacterial, fungal and nematode communities along an Antarctic environmental gradient FEMS Microbiol Ecol 59 436-451

Yi H and Chun J (2006) Flavobacterium weaverense sp. nov. and Flavobacterium segetis sp. nov., novel psychrophiles isolated from the Antarctic Int J Syst Evol Microbiol 56 1239-1244

Yu Y, Li H R, Zeng Y X and Chen B (2011) Bacterial diversity and bioprospecting for cold-active hydrolytic enzymes from culturable bacteria associated with sediment from Nella Fjord, Eastern Antarctica Mar Drugs 9 184-195

Zhao J, Yang N and Zeng R (2008) Phylogenetic analysis of type I polyketide synthase and nonribosomal peptide synthetase genes in Antarctic sediment Extremophiles 12 97-105. 